\title{
A mathematical model for thermal single-phase flow and reactive transport in fractured porous media
}

\author{
Alessio Fumagalli \\ Anna Scotti \\ MOX - Dipartimento di Matematica "F. Brioschi", Politecnico di Milano, via \\ Bonardi 9, 20133 Milan, Italy.
}

\begin{abstract}
In this paper we present a mathematical model and a numerical workflow for the simulation of a thermal single-phase flow with reactive transport in porous media, in the presence of fractures. The latter are thin regions which might behave as high or low permeability channels depending on their physical parameters, and are thus of paramount importance in underground flow problems. Chemical reactions may alter the local properties of the porous media as well as the fracture walls, changing the flow path and possibly occluding some portions of the fractures or zones in the porous media. To solve numerically the coupled problem we propose a temporal splitting scheme so that the equations describing each physical process are solved sequentially. Numerical tests shows the accuracy of the proposed model and the ability to capture complex phenomena, where one or multiple fractures are present.
\end{abstract}

\section{Introduction}

The presence of fractures has an impact on subsurface flows at all scales: flow tends to focus along highly permeable fractures, which can create shortcuts in the domain, or, in the case of cemented or low permeable fractures, they might create barriers in the domain. In the context of reactive transport fractures can be responsible for fast transport of fluid with different chemical composition with respect to the surrounding matrix: this occurs for instance in geothermal reservoirs where water with different salinity, solutes and temperature is injected in the subsurface. These differences in composition and temperature can trigger transformations such as mineral precipitation, dissolution or replacement, with an impact on porosity and fracture aperture. The effective exploitation of the geothermal system can be jeopardized by such phenomena.

Because of their thickness or aperture, fractures are usually represented as lower dimensional objects and new equations along with interface conditions with the surrounding porous media are derived. This procedure is usually referred to as model reduction and the resulting model is named mixed-dimensional or hybrid-dimensional problem. Seminal works dealing with singlephase flow are for example [6, 5, 27, 7, 52. During the years new models have been developed based on this idea, in particular for multi-phase flow [39, 4], transport [61, 21], and faults flow 62, 28, 35. The geometrical complexity of the fracture networks requires to handle in an accurate way also the intersection between them, indeed the intersection may have different physical parameters than the incident fractures. In this case new models have been derived where the intersections is part of the problem, see for example [32, 18, 60. In the special case of high speed circulation of the liquid in the fractures, the Darcy model may be not appropriate. Thus several authors proposed a new model based on Forchheimer or even more advanced flow 
model. Refer to $[34,53,54,50,3$. Finally, we refer to 16 for a more detailed review on different strategies to handle the complex problem of fractured porous medium.

The numerical solution of these problems is challenging due to several aspects, in fact the fracture networks may pose severe constraints in the grid generation resulting in poor quality and too many elements. Since this work is more focused on the modelling side, we refer to the main works that dealt with different classes of numerical schemes: classical mixed finite elements [52, hybrid high-order [20, discontinuous Galerkin [8, mimetic finite differences [9, extended finite elements [32, 41, 60, 31, virtual element method 36, 37, and references therein. Important benchmark studies to validate the effectiveness of the numerical schemes are [23, 30, 38, 15. Finally, a unified approach for numerical frameworks to solve such problems is presented in [55].

The aim of our work is to propose a model to account explicitly for the presence of fractures and their impact on the flow, temperature, transport and reactions. The equations describing flow and transport are thus a coupled system of mixed-dimensional PDEs which will be approximated by means of lowest order mixed finite elements or mixed virtual elements, depending on the geometrical complexity of the computational grid. We will consider a simple model for mineral precipitation and dissolution following the model presented, among others, in [2. To avoid the occurrence of negative concentrations and oscillations when the amount of precipitate approaches zero we adopt an event detection/location strategy to detect the discontinuity in the ODE describing the reaction part, which is, for this reason, split from advection and diffusion by means of a first-order operator splitting. Several numerical examples will show the validity of our approach for increasing level of geometrical difficulty of the fracture network.

The paper is organized as follow. In Section 2 we introduce the mathematical model to describe fluid flow, heat transport, and solute transport with chemical reactions in porous media. The latter are particularized in Section 3 The mixed-dimensional problem to describe the physical processes in the fractures is discussed in Section 4. Section 5 presents the discretization considered to approximate the models, in particular a splitting scheme is detailed that allows for a sequential resolution of each physical process involved in the simulation. In Section 6 we run different examples to show the validity and accuracy of the proposed approach. Finally, Section 7 is devoted to the conclusions.

\section{Model in the porous media}

In this section we describe the mathematical model for our problem. The physical processes are described separately but coupled together by suitable constitutive relations. We first focus on the model in the porous matrix, leaving for a subsequent section the introduction of the reduced model to include the fracture effects. First, we introduce the Darcy flow in Subsection 2.1 followed by the heat equation in Subsection 2.2. The models for the solute and precipitate are presented in Subsection 2.3. and the section concludes with the constitutive relations in Subsection 2.4

The porous media occupies the domain $\Omega \subset \mathbb{R}^{n}$, with $n=2$ or 3 , with Lipschitz continuous external boundary $\partial \Omega$. The latter has been divided into two disjoint, possibly empty, subsets $\partial_{e} \Omega$ and $\partial_{n} \Omega$, such that $\overline{\partial \Omega}=\overline{\partial_{e} \Omega} \cup \overline{\partial_{n} \Omega}$ and $\partial_{e}^{\circ} \Omega \cap \partial_{u}^{\circ} \Omega=\emptyset$. For simplicity we assume that $\partial_{n} \Omega \neq \emptyset$. The outward unit normal of $\partial \Omega$ is indicated as $\boldsymbol{n}_{\partial \Omega}$, see Figure 1 . Finally, the final time is indicated as $T>0$.

It is important to note that, since the flow equation is the main driving force of the process, the boundary conditions of the other problems are conforming with the ones imposed on the flow problem.

In the subsequent parts we will explicitly indicate the units of measure of each variable and 


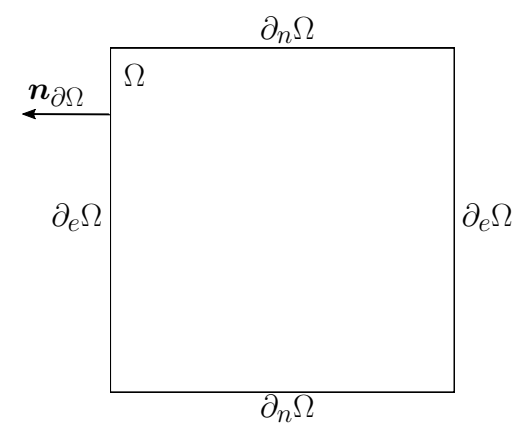

Figure 1: Example of porous media with some nomenclature considered.

coefficient. We will make use of the notation $1\left[\mathrm{~m}_{\phi}^{3}\right]$ to be the unit cubic meter of pore space, defined as $\mathrm{m}_{\phi}^{3}=\phi \mathrm{m}^{3}$ with $\phi$ the porosity.

General references for the following models are, for example, [10, 11, 46, 56].

\subsection{Darcy flow model}

We consider a Darcy model to describe the flow of the water in the underground. We are interested in the evolution of the Darcy velocity $\boldsymbol{q}$ in $\left[\mathrm{m} \mathrm{s}^{-1}\right]$ and pressure $p$ in $[\mathrm{Pa}]$ described by the system

$$
\begin{aligned}
& \mu \boldsymbol{q}+k(\phi) \nabla p=\mathbf{0} \\
& \partial_{t} \phi+\nabla \cdot \boldsymbol{q}+f=0
\end{aligned} \quad \text { in } \Omega \times(0, T) .
$$

The scalar source or sink term is denoted by $f$ in $\left[\mathrm{s}^{-1}\right]$. To keep the model simpler, we assume that the water viscosity $\mu$ in $[\mathrm{Pas}]$ can be considered constant. The permeability $k$ in $\left[\mathrm{m}^{2}\right]$ is a symmetric, isotropic and positive definite tensor which depends on the porosity $\phi$ in $\left[\mathrm{m}_{\phi}^{3} \mathrm{~m}^{-3}\right]$ which in turn, as we will see in a subsequent model in Subsection 2.4 depends on the precipitate concentration. Thus also the porosity is a variable of the previous system. We assume that $\phi \in[0,1]$.

Boundary conditions are coupled to 1 a to close the system. In particular, we have

$$
\begin{array}{ll}
\operatorname{tr} \boldsymbol{q} \cdot \boldsymbol{n}_{\partial \Omega}=q_{\partial \Omega} & \text { on } \partial_{e} \Omega \\
\operatorname{tr} p=p_{\partial \Omega} & \text { on } \partial_{n} \Omega
\end{array}
$$

where tr denotes an abstract trace operator, $p_{\partial \Omega}$ in $[\mathrm{Pa}]$ and $q_{\partial \Omega}$ in $\left[\mathrm{ms}^{-1}\right]$ are the pressure and normal flux given data. System (1) forms the Darcy flow problem.

\section{$2.2 \quad$ Heat model}

The heat equation models thermal conduction (Fourier's law) and convection of heat in the porous media. A complete model should consider two sets of equations: one for the rock matrix and one for the water, coupled with a suitable transfer function. However we assume local thermal equilibrium, meaning that the rock matrix and water are in thermal equilibrium so we can use only one common set of primary variables to describe the process. The temperature field is indicated as $\theta$ in $[\mathrm{K}]$ and its evolution is described by

$$
\begin{array}{ll}
\boldsymbol{\tau}-\rho_{w} c_{w} \boldsymbol{q} \theta+\lambda(\phi) \nabla \theta=\mathbf{0} & \text { in } \Omega \times(0, T), \\
\partial_{t}[c(\phi) \theta]+\nabla \cdot \boldsymbol{\tau}+j=0 &
\end{array}
$$


where $\boldsymbol{\tau}$ in $\left[\mathrm{J} \mathrm{m}^{-2} \mathrm{~s}^{-1}\right]$ is the total heat flux, $c$ in $\left[\mathrm{J} \mathrm{m}^{-3} \mathrm{~K}^{-1}\right]$ is the effective thermal capacity which is defined as the porosity weighted average between the water $c_{w}$ and solid $c_{s}$ specific thermal capacity, both in $\left[\mathrm{J} \mathrm{kg}^{-1} \mathrm{~K}^{-1}\right]$. We have thus the expression of $c$ given by

$$
c(\phi)=\phi \rho_{w} c_{w}+(1-\phi) \rho_{s} c_{s} .
$$

$\rho_{w}$ and $\rho_{s}$ are the densities, both in $\left[\mathrm{kg} \mathrm{m}^{-3}\right]$, of the water and solid phase respectively. Finally, $\lambda$ is the effective thermal conductivity measured in $\left[\mathrm{W} \mathrm{m}^{-1} \mathrm{~K}^{-1}\right]$. For simplicity, we assume that the densities and the specific thermal capacities are given and constant. Following [22, being the porous media saturated with water, we model the effective thermal conductivity as

$$
\lambda(\phi)=\lambda_{w}^{\phi} \lambda_{s}^{1-\phi},
$$

where $\lambda_{s}$ and $\lambda_{s}$ are water and solid thermal conductivity, both in $\left[\mathrm{W} \mathrm{m}^{-1} \mathrm{~K}^{-1}\right]$, and for simplicity assumed to be constant. Finally, $j$ in $\left[\mathrm{J} \mathrm{m}^{-3} \mathrm{~s}^{-1}\right]$ models a source or sink of heat in the system.

In addition to system 2a), we consider suitable boundary and initial conditions,

$$
\begin{array}{ll}
\operatorname{tr} \boldsymbol{\tau} \cdot \boldsymbol{n}_{\partial \Omega}=\tau_{\partial \Omega} & \text { on } \partial_{n} \Omega \times(0, T) \\
\operatorname{tr} \theta=\theta_{\partial \Omega} & \text { on } \partial_{e} \Omega \times(0, T), \\
\theta(t=0)=\theta_{0} & \text { in } \Omega \times\{0\}
\end{array}
$$

where $\tau_{\partial \Omega}$ in $\left[\mathrm{J} \mathrm{m}^{-2} \mathrm{~s}^{-1}\right]$ and $\theta_{\partial \Omega}$ in $[\mathrm{K}]$ are suitable boundary data for the heat flux and temperature, respectively. Finally, $\theta_{0}$ in $[\mathrm{K}]$ is the initial condition for the temperature. System (2) forms the heat problem.

\subsection{Solute and precipitate model}

We consider the passive scalar model to describe the evolution of the solute $u$ in $\left[\mathrm{mol} \mathrm{m}_{\phi}^{-3}\right]$ in the porous medium. Note that solute concentration is expressed in terms of moles per unit pore volume, so we should have $u \geq 0$. The system is written as

$$
\begin{array}{ll}
\boldsymbol{\chi}-\boldsymbol{q} u+\phi d \nabla u=\mathbf{0} & \text { in } \Omega \times(0, T), \\
\partial_{t}(\phi u)+\nabla \cdot \boldsymbol{\chi}+\phi r_{w}(u, w ; \theta)=0 &
\end{array}
$$

with $\chi$ in $\left[\mathrm{mol} \mathrm{m}^{-2} \mathrm{~s}^{-1}\right]$ the total flux given by a combination of the advective field and Fick's law. The symmetric, isotropic and positive definite tensor $d$ in $\left[\mathrm{m}^{2} \mathrm{~s}^{-1}\right]$ represents the molecular diffusivity of $u$ in the water and $r_{w}$ in $\left[\mathrm{mol} \mathrm{m}_{\phi}^{-3} \mathrm{~s}^{-1}\right]$ is a reaction term which involves also the precipitate $w$, as well as the temperature $\theta$ and will be detailed later on.

To model the concentration of the precipitate $w \geq 0$, expressed in $\left[\mathrm{mol} \mathrm{m}_{\phi}^{-3}\right]$, we consider the following ordinary differential equation which models its evolution in time

$$
\partial_{t}(\phi w)-\phi r_{w}(u, w ; \theta)=0 \quad \text { in } \Omega \times(0, T) .
$$

The actual expression of $r_{w}$ depends on several aspects, see Section 3 for a more detailed discussion.

Boundary and initial conditions are supplied to equations $3 \mathrm{a}$ and $3 \mathrm{~b}$ as

$$
\begin{array}{ll}
\operatorname{tr} \boldsymbol{\chi} \cdot \boldsymbol{n}_{\partial \Omega}=\chi_{\partial \Omega} & \text { on } \partial_{e} \Omega \times(0, T) \\
\operatorname{tr} u=u_{\partial \Omega} & \text { on } \partial_{n} \Omega \times(0, T), \\
u(t=0)=u_{0} & \text { in } \Omega \times\{0\} \\
w(t=0)=w_{0} & \text { in } \Omega \times\{0\}
\end{array},
$$




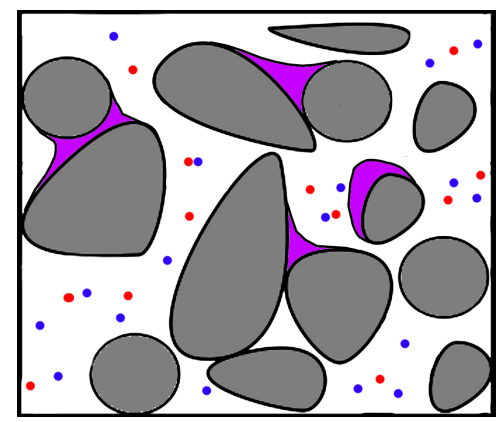

Figure 2: Graphical representation that shows the two solutes (blue and red dots) reacting and forming the precipitate (violet) around the grains.

where the values of $\chi_{\partial \Omega}$ in $\left[\mathrm{mol} \mathrm{m}^{-2} \mathrm{~s}^{-1}\right]$ and $u_{\partial \Omega}$ in $\left[\mathrm{mol} \mathrm{m}_{\phi}^{-3}\right]$ are the normal component of total flux and solute data imposed at the boundary $\partial \Omega$. Finally, $u_{0}$ in $\left[\mathrm{mol} \mathrm{m}_{\phi}^{-3}\right]$ and $w_{0}$ in $\left[\mathrm{mol} \mathrm{m}_{\phi}^{-3}\right]$ specify the initial conditions for both the solute and precipitate. System (3) forms the solute and precipitate problem.

\subsection{Permeability and porosity model}

We consider a Kozeny-type relationship [10] to link the permeability with the porosity, namely

$$
k(\phi)=k_{0} \frac{\phi^{2}}{\phi_{0}^{2}}
$$

where $k_{0}$ in $\left[\mathrm{m}^{2}\right]$ and $\phi_{0}$ in $\left[\mathrm{m}_{\phi}^{3} \mathrm{~m}^{-3}\right]$ are the given reference permeability and porosity, respectively. Other and more general relationships between $k$ and $\phi$ are possible. Finally, the model that links the porosity $\phi$ to the precipitate $w$ is given by the following ODE,

$$
\begin{array}{ll}
\partial_{t} \phi+\eta_{\Omega} \phi \partial_{t} w=0 & \text { in } \Omega \times(0, T) \\
\phi(t=0)=\phi_{0} & \text { in } \Omega \times\{0\}
\end{array},
$$

where $\eta_{\Omega}$ in $\left[\mathrm{m}_{\phi}^{3} \mathrm{~mol}^{-1}\right]$ is a proportionality parameter associated with molar volume of the mineral, see [63], that determines the rate of deposition of the solute around the grains as shown in Figure 2

\subsection{Complete model}

The model to describe flow, heat conduction and convection, precipitation and dissolution of the chemical species as well as permeability and porosity alteration is given by the system of equations (1), (2), (3), (4) and (5). The system is fully coupled, non-linear and possibly non-smooth due to the discontinuous reaction rate.

\subsection{Non-dimensional equations}

We now derive the non-dimensional version of the solute equation to identify some non-dimensional numbers that could characterize the solution behaviour. Let us define some reference quantities: $L$ for length, $Q$ for velocity, $\phi_{\Omega, 0}$ for porosity, $u_{e}$ for molar concentration. Let us denote with.$^{\prime}$ 
nondimensional quantities, so that $x^{\prime}=x / L, t^{\prime}=t U / L$, so that $\partial_{t}=\partial t^{\prime} U / L$ and $\partial x=\partial x^{\prime} 1 / L$. In primary form, the solute equation $(3 \mathrm{a})$ can be rewritten as

$$
\partial_{t^{\prime}}\left(u^{\prime} \phi\right)+\nabla^{\prime} \cdot\left(u^{\prime} \boldsymbol{q}^{\prime}\right)-\frac{D}{L Q} \nabla^{\prime} \cdot\left(\phi \nabla^{\prime} u^{\prime}\right)=-\frac{\phi L}{Q} \lambda r_{w}^{\prime} .
$$

The above equation simplifies if we consider constant porosity, $\phi=\phi_{0}$.

$$
\partial_{t^{\prime}} u^{\prime}+\frac{1}{\phi_{0}} \nabla^{\prime} \cdot\left(u^{\prime} \boldsymbol{q}^{\prime}\right)-\frac{D}{L Q} \nabla^{\prime} \cdot\left(\nabla^{\prime} u^{\prime}\right)=-\frac{L \lambda}{Q} r_{w}^{\prime} .
$$

Note that we can define a "Reynolds number" as $\mathbb{R} e=L Q \phi_{0} / D$ and a Damköhler number $\mathbb{D} a=L \lambda \phi_{0} / Q$. A large $\mathbb{D} a$ corresponds to fast reactions with respect to advection, while a small $\mathbb{D} a$ corresponds to fast advection with respect to reaction speed.

\section{Chemical model}

Our idealized model for chemistry considers two reactions: precipitation and dissolution, which can be written as

$$
\begin{aligned}
& \alpha U+\beta^{+} V \rightarrow W+\beta^{-} V \\
& W+\beta^{-} V \rightarrow \alpha U+\beta^{+} V
\end{aligned}
$$

where $U$ and $V$ are two solutes (ions) that can precipitate to form a solid (salt) $W$, and $\alpha$ and $\beta^{ \pm}$ are integer stoichiometric coefficients. According to the mass action law [45], the precipitation rate depends on the rate $\lambda^{+}$, which is the reaction constant for (6), and on the concentrations of the two ions raised to the power indicated by the stoichiometric coefficients $\alpha$ and $\beta^{+}$, conversely, the rate of dissolution depends only on the reactant $v$ if $\beta^{-}$is greater than zero. The latter being the reaction constant for (7). In our model, these two coefficients might depend on the temperature $\theta$ of the system $\lambda^{ \pm}=\lambda^{ \pm}(\theta)$. The net rate of precipitation is thus a function of $u$ and $v$, and it is given by

$$
r_{w}(u, v ; \theta)=\lambda^{+}(\theta) u^{\alpha} v^{\beta^{+}}-\lambda^{-}(\theta) v^{\beta^{-}}
$$

where $u, v$ are the molar concentrations in $\left[\mathrm{mol} \mathrm{m}^{-3}\right]$ of $U$ and $V$, respectively. If we consider this simplified set of reactions

$$
\begin{aligned}
& \alpha U+\beta V \rightarrow W \\
& W \rightarrow \alpha U+\beta V
\end{aligned}
$$

the net precipitation rate becomes $r_{w}(u, v ; \theta)=\lambda^{+}(\theta) u^{\alpha} v^{\beta}-\lambda^{-}(\theta)$, i.e. we have a dissolution rate $\lambda^{-}$that is independent from $u$ and $v$ since it is established in literature that the activity of a pure crystalline solid is a constant [51]. At equilibrium the net rate of precipitation is zero, yielding

$$
\lambda^{+}(\theta) u_{e}^{\alpha} v_{e}^{\beta}=\lambda^{-}(\theta),
$$

where $u_{e}$ and $v_{e}$ are the molar concentrations of $u$ and $v$ at equilibrium, respectively. The net precipitation rate can be rewritten as

$$
r_{w}(u, v ; \theta)=\lambda^{-}(\theta)\left(\frac{u^{\alpha} v^{\beta}}{u_{e}^{\alpha} v_{e}^{\beta}}-1\right) .
$$




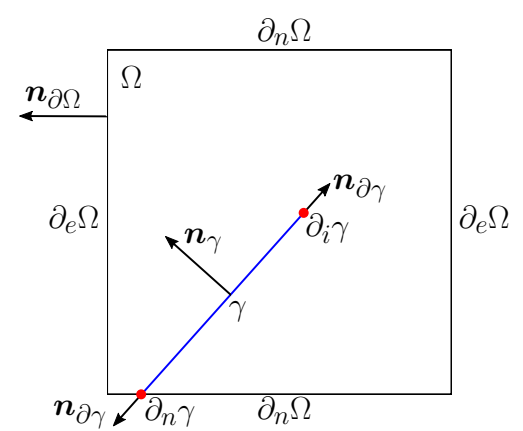

Figure 3: Example of fractured porous media with some nomenclature considered.

We further assume that there is electrical equilibrium in the system, i.e. the number of cations equals the number of anions, $u=v$ and we can consider only one of the two variables to describe the reaction rate. For example, in the special case of $\alpha=\beta=1$ the reaction becomes $U+V \leftrightarrow W$ and the reaction rate can be written as

$$
r_{w}(u ; \theta)=\lambda^{-}(\theta)\left[\left(\frac{u}{u_{e}}\right)^{2}-1\right]
$$

In our work we consider problems with a reaction rate is given by a function $r$ which depends on one of the two solute

$$
r_{w}(u ; \theta)=\lambda^{-}(\theta)[r(u)-1]
$$

Finally, note that the rate of dissolution does not depend on the concentration of the solid salt $W$, in other words it does not vanish when $w=0$. This condition must be enforced in the model, so that dissolution stops only when $w \leq 0$ and the net precipitation rate is negative, i.e. when the precipitate is no longer present but the solute concentration is such that we should have dissolution. This can be summarized in the following more general expression

$$
r_{w}(u, w ; \theta)=\lambda^{-}(\theta)\{\max [r(u)-1,0]+H(w) \min [r(u)-1,0]\},
$$

where $H$ is the Heaviside function. The latter expression of $r_{w}$ is the one used in this work.

\section{Model in the fracture}

We here introduce a mixed-dimensional model to approximate the problem described in the previous sections in the presence of fractures. We start with the simplified assumption of a single fracture $\gamma$ cutting the domain as shown in Figure 3 . The case of multiple intersecting fractures will be described later. Since a fracture is an equi-dimensional region, i.e. 2D or 3D region with a small thickness, we adopt the model reduction strategy to reduce the geometrical complexity and we approximate it as a one co-dimensional object. The fracture aperture $\epsilon_{\gamma}$ in $[\mathrm{m}]$ will become a model parameter and not a geometrical constraint, which, in our case, is even more beneficial since it might change during the simulation. For more references on this approach see [52, 53, 33, 48, 62, 54, 59, 41, 23, 60, 37, 55, 61, 20, 3, 16, 42, to name a few.

We assume that the fracture is open without any presence of infilling porous material, getting unitary porosity in the fracture itself. So we get that $\mathrm{m}_{\phi}^{3}=\mathrm{m}^{3}$ in the fracture, i.e. the pore volume 
and total volume are equivalent. Now the role played by $\phi$ in the surrounding porous medium is given by the aperture $\epsilon_{\gamma}$.

The fracture $\gamma$ is a non self-intersecting piece-wise $C^{2}$ curve (if $n=2$ ) or surface (if $n=3$ ). We indicate with $\partial \gamma$ the boundary of $\gamma$, which can be divided into three disjoint, possible empty, parts: $\partial_{e} \gamma, \partial_{n} \gamma$, and $\partial_{i} \gamma$. The latter is the part of $\partial \gamma$ internal to the porous media. We clearly have $\overline{\partial \gamma}=\overline{\partial_{e} \gamma} \cup \overline{\partial_{n} \gamma} \cup \overline{\partial_{i} \gamma}$ and also that $\partial_{*}^{\circ} \gamma \cap \partial_{*^{\prime}}^{\circ} \gamma=\emptyset$ for any combination of non-equal elements in $*, *^{\prime} \in\{e, n, i\}$. We can define an outward unit normal to $\partial \gamma$, which is tangent to $\gamma$ itself and orthogonal to $\partial \gamma$ named $\boldsymbol{n}_{\partial \gamma}$.

At the interface with the surrounding medium, the fracture has two different sides $\gamma_{+}$and $\gamma_{-}$with associated normal vectors $\boldsymbol{n}_{+}$and $\boldsymbol{n}_{-}$. We assign a unique normal $\boldsymbol{n}_{\gamma}$ to the fracture which is associated with both fracture sides (i.e., $\boldsymbol{n}_{\gamma}=\boldsymbol{n}_{\gamma,+}$ on $\gamma_{+}$and $\boldsymbol{n}_{\gamma}=-\boldsymbol{n}_{\gamma,-}$ on $\gamma_{-}$). See Figure 3 as an example.

\subsection{Reduced variables}

Variables and data associated with the fracture will be denoted with a subscript $\gamma$, while we use a subscript $\Omega$ to indicate variables and data in the surrounding porous media. We introduce the fracture vector variables which come from the integration over each normal section of the fracture of the corresponding equi-dimensional variables, for $\boldsymbol{x} \in \gamma$ the curvilinear abscissa, as

$$
\boldsymbol{q}_{\gamma}(\boldsymbol{x}):=\int_{\epsilon_{\gamma}(\boldsymbol{x})} T(\boldsymbol{x}) \boldsymbol{q}(\boldsymbol{x}, s) d s \quad \boldsymbol{\tau}_{\gamma}(\boldsymbol{x}):=\int_{\epsilon_{\gamma}(\boldsymbol{x})} T(\boldsymbol{x}) \boldsymbol{\tau}(\boldsymbol{x}, s) d s \quad \boldsymbol{\chi}_{\gamma}(\boldsymbol{x}):=\int_{\epsilon_{\gamma}(\boldsymbol{x})} T(\boldsymbol{x}) \chi(\boldsymbol{x}, s) d s
$$

with $T:=I-N$ and $N:=\boldsymbol{n}_{\gamma} \otimes \boldsymbol{n}_{\gamma}$ the tangential and normal projection matrices, respectively. The unit of measure of the previous variables are: $\boldsymbol{q}_{\gamma}$ in $\left[\mathrm{m}^{2} \mathrm{~s}^{-1}\right], \boldsymbol{\tau}_{\gamma}$ in $\left[\mathrm{J} \mathrm{m}^{-1} \mathrm{~s}^{-1}\right]$, and $\boldsymbol{\chi}_{\gamma}$ in $\left[\mathrm{mol} \mathrm{m}^{-1} \mathrm{~s}^{-1}\right]$. Moreover, the reduced scalar fields are defined from their respective equidimensional variable as the average for each section,

$$
\begin{aligned}
p_{\gamma}(\boldsymbol{x}) & :=\frac{1}{\epsilon_{\gamma}(\boldsymbol{x})} \int_{\epsilon_{\gamma}(\boldsymbol{x})} p(\boldsymbol{x}, s) d s \quad \theta_{\gamma}(\boldsymbol{x}):=\frac{1}{\epsilon_{\gamma}(\boldsymbol{x})} \int_{\epsilon_{\gamma}(\boldsymbol{x})} \theta(\boldsymbol{x}, s) d s \\
u_{\gamma}(\boldsymbol{x}) & :=\frac{1}{\epsilon_{\gamma}(\boldsymbol{x})} \int_{\epsilon_{\gamma}(\boldsymbol{x})} u(\boldsymbol{x}, s) d s \quad w_{\gamma}(\boldsymbol{x}):=\frac{1}{\epsilon_{\gamma}(\boldsymbol{x})} \int_{\epsilon_{\gamma}(\boldsymbol{x})} w(\boldsymbol{x}, s) d s .
\end{aligned}
$$

In this case, the units of measures are the same as the original variables: $p_{\gamma}$ in $[\mathrm{Pa}], \theta_{\gamma}$ in $[\mathrm{K}]$, $u_{\gamma}$ in $\left[\mathrm{mol} \mathrm{m}^{-3}\right]$, and $w_{\gamma}$ in $\left[\mathrm{mol} \mathrm{m}^{-3}\right]$. With an abuse in notation, we introduce the flux-based variable compounds as

$$
\boldsymbol{q}:=\left(\boldsymbol{q}_{\Omega}, \boldsymbol{q}_{\gamma}\right) \quad \boldsymbol{\tau}:=\left(\boldsymbol{\tau}_{\Omega}, \boldsymbol{\tau}_{\gamma}\right) \quad \chi:=\left(\chi_{\Omega}, \chi_{\gamma}\right)
$$

even if that the units of measure of each compound are heterogeneous.

When the nabla operator is applied to a fracture variable, we implicitly assume that it is defined on the tangential space of the fracture itself, i.e. $\nabla \cdot \boldsymbol{\nu}_{\gamma}:=T: \tilde{\nabla} \boldsymbol{\nu}_{\gamma}, \tilde{\nabla}$ being the standard gradient and $\boldsymbol{\nu}_{\gamma}$ a regular enough vector function defined on $\gamma$. Analogously for the gradient of a fracture variable, which is defined as $\nabla \nu_{\gamma}:=T \tilde{\nabla} \nu_{\gamma}$ with $\nu_{\gamma}$ a regular enough scalar function defined on $\gamma$. The conservation operators or mixed-dimensional divergences are defined on the compounds and are given by

$$
\nabla_{\Omega} \cdot \boldsymbol{\nu}:=\nabla \cdot \boldsymbol{\nu}_{\Omega} \quad \text { and } \quad \nabla_{\gamma} \cdot \boldsymbol{\nu}:=\nabla \cdot \boldsymbol{\nu}_{\gamma}-\operatorname{tr} \boldsymbol{\nu}_{\Omega} \cdot \boldsymbol{n}_{\gamma}
$$

with $\boldsymbol{\nu}=\left(\boldsymbol{\nu}_{\Omega}, \boldsymbol{\nu}_{\gamma}\right)$ a generic compound of vector variables. The $\nabla_{\gamma}$. considers also the contribution from the surrounding porous media into the fracture as flux exchange, and tr indicates the trace operator from $\Omega$ to each side of the fracture, $\gamma_{+}$and $\gamma_{-}$, viewed from $\Omega$. 
Following the idea in [52, we require that the coefficients associated to the diffusion coefficients $k$ and $d$ can be decomposed as

$$
k=\kappa N+k_{\gamma} T \quad \text { and } \quad d=\delta N+d_{\gamma} T,
$$

where the first relation implies that the permeability $k$ can be decomposed in its normal $\kappa$ (in $\left.\left[\mathrm{m}^{2}\right]\right)$ and tangential $k_{\gamma}\left(\right.$ in $\left.\left[\mathrm{m}^{2}\right]\right)$ parts with respect to the fracture geometry. The same applies for molecular diffusivity, while the effective thermal conductivity (2c) is a scalar value, so it does not need this decomposition. Note that for some specific cases the value of the normal and tangential component might be equal, however to keep a more general setting we use different symbols.

\subsection{Reduced Darcy flow model}

The reduced model for the Darcy flow describes the evolution of the reduced Darcy velocity $\boldsymbol{q}_{\gamma}$ and pressure $p_{\gamma}$ in the fracture, and reads

$$
\begin{array}{ll}
\mu \boldsymbol{q}_{\gamma}+\epsilon_{\gamma} k_{\gamma}\left(\epsilon_{\gamma}\right) \nabla p_{\gamma}=\mathbf{0} & \text { in } \gamma \times(0, T), \\
\partial_{t} \epsilon_{\gamma}+\nabla_{\gamma} \cdot \boldsymbol{q}+\epsilon_{\gamma} f_{\gamma}=0 &
\end{array}
$$

where the reduced source or sink term $f_{\gamma}\left[\mathrm{s}^{-1}\right]$ is computed as $f_{\gamma}(\boldsymbol{x}):=\epsilon_{\gamma}^{-1}(\boldsymbol{x}) \int_{\epsilon_{\gamma}(\boldsymbol{x})} f(\boldsymbol{x}, s) d s$. Following lubrication theory, the fracture tangential permeability $k_{\gamma}$ is expressed as a function of the aperture, as described in more detail in Subsection 4.5. Due to the introduction of the mixeddimensional divergences the form of $10 \mathrm{a}$ is similar to (1a). At the fracture-matrix interface we consider a discrete version of Darcy's law in the normal direction, see for example [52, which is given by

$$
\mu \epsilon_{\gamma} \operatorname{tr} \boldsymbol{q}_{\Omega} \cdot \boldsymbol{n}_{\gamma}+\kappa_{\gamma}\left(\epsilon_{\gamma}\right)\left(p_{\gamma}-\operatorname{tr} p_{\Omega}\right)=0 \quad \text { on } \gamma \times(0, T) .
$$

The latter relation models the fact that the flux exchange between the fracture and the surrounding porous media is related to the pressure jump via $\kappa_{\gamma}$. This parameter, defined by (13), is related to the aperture with a power law of exponent greater than one. Thus, if the aperture goes to zero the flux exchange vanishes and the fracture and porous media become decoupled.

Finally, we need to supply boundary conditions also to the fracture tips. In particular, we distinguish between immersed tips and tips touching the domain boundary. In the first case, the so-called tip conditions are considered while in the latter case we inherit the boundary conditions from the equi-dimensional problem. In formula

$$
\begin{array}{ll}
\operatorname{tr} \boldsymbol{q}_{\gamma} \cdot \boldsymbol{n}_{\partial \gamma}=q_{\partial \gamma} & \text { on } \partial_{e} \gamma \times(0, T) \\
\operatorname{tr} p_{\gamma}=p_{\partial \gamma} & \text { on } \partial_{n} \gamma \times(0, T), \\
\operatorname{tr} \boldsymbol{q}_{\gamma} \cdot \boldsymbol{n}_{\partial \gamma}=0 & \text { on } \partial_{i} \gamma \times(0, T)
\end{array}
$$

with $q_{\partial \gamma}$ in $\left[\mathrm{m}^{2} \mathrm{~s}^{-1}\right]$ and $p_{\partial \gamma}$ in $[\mathrm{Pa}]$ are the given flux and pressure at the fracture boundary. The last condition is the tip condition which imposes no flow, see [7]. System (10] is the reduced Darcy flow problem.

\subsection{Reduced heat model}

As we did in Subsection 2.2, we assume also in the fracture local thermal equilibrium. However, in a fracture we can reach high speed circulation of water which might invalidate this assumption. 
To keep the presentation simple, we leave this case for future investigations. Following the idea in [40, the heat equation which models the thermal flux $\boldsymbol{\tau}_{\gamma}$ and temperature $\theta_{\gamma}$ in $\gamma$ is written as

$$
\begin{array}{ll}
\boldsymbol{\tau}_{\gamma}-\rho_{w} c_{w} \boldsymbol{q}_{\gamma} \theta_{\gamma}+\epsilon_{\gamma} \lambda_{w} \nabla \theta_{\gamma}=\mathbf{0} \\
\rho_{w} c_{w} \partial_{t}\left(\epsilon_{\gamma} \theta_{\gamma}\right)+\nabla_{\gamma} \cdot \boldsymbol{\tau}+j_{\gamma}=0
\end{array} \quad \text { in } \gamma \times(0, T)
$$

Being the fracture open, the effective thermal capacity and conductivity are simplified to $\lambda=\lambda_{w}$ as well as $c=\rho_{w} c_{w}$. The source term $j_{\gamma}$ in $\left[\mathrm{J} \mathrm{m}^{-3} \mathrm{~s}^{-1}\right]$ is computed as $j_{\gamma}(\boldsymbol{x}):=\epsilon_{\gamma}^{-1}(\boldsymbol{x}) \int_{\epsilon_{\gamma}(\boldsymbol{x})} j(\boldsymbol{x}, s) d s$. At the interface between the fracture and surrounding porous media the coupling conditions are

$$
\epsilon_{\gamma}\left(\operatorname{tr} \boldsymbol{\tau}_{\Omega} \cdot \boldsymbol{n}_{\gamma}-\rho_{w} c_{w} \operatorname{tr} \boldsymbol{q}_{\Omega} \cdot \boldsymbol{n}_{\gamma} \operatorname{tr} \theta_{\Omega}\right)+\lambda_{w}\left(\theta_{\gamma}-\operatorname{tr} \theta_{\Omega}\right)=0 \quad \text { on } \gamma \times(0, T) .
$$

The boundary and initial conditions for the reduced heat equation are inherit from the equidimensional problem and they are given by

$$
\begin{array}{ll}
\operatorname{tr} \boldsymbol{\tau}_{\gamma} \cdot \boldsymbol{n}_{\partial \gamma}=\tau_{\partial \gamma} & \text { on } \partial_{e} \gamma \times(0, T) \\
\operatorname{tr} \theta_{\gamma}=\theta_{\partial \gamma} & \text { on } \partial_{n} \gamma \times(0, T) \\
\operatorname{tr} \boldsymbol{\tau}_{\gamma} \cdot \boldsymbol{n}_{\partial \gamma}=0 & \text { on } \partial_{i} \gamma \times(0, T) \\
\theta_{\gamma}(t=0)=\theta_{\gamma, 0} & \text { in } \gamma \times\{0\}
\end{array}
$$

where $\tau_{\partial \gamma}$ in $\left[\mathrm{J} \mathrm{m}^{-1} \mathrm{~s}^{-1}\right]$ and $\theta_{\partial \gamma}$ in $[\mathrm{K}]$ are the thermal flux and temperature boundary data, respectively, and the third condition is the internal tip condition. The data $\theta_{\gamma, 0}$ in $[\mathrm{K}]$ is the initial temperature distribution in the fracture. System (11) is the reduced system for temperature.

\subsection{Reduced solute and precipitate model}

The reduced model that describes the evolution of the solute $u_{\gamma}$ and its flux $\chi_{\gamma}$ can be written in the following way

$$
\begin{aligned}
& \boldsymbol{\chi}_{\gamma}-\boldsymbol{q}_{\gamma} u_{\gamma}+\epsilon_{\gamma} d_{\gamma} \nabla u_{\gamma}=\mathbf{0} \\
& \partial_{t}\left(\epsilon_{\gamma} u_{\gamma}\right)+\nabla_{\gamma} \cdot \chi+\epsilon_{\gamma} r_{w}\left(u_{\gamma}, w_{\gamma} ; \theta_{\gamma}\right)=0
\end{aligned} \quad \text { in } \gamma \times(0, T) .
$$

Now the reaction term $r_{w}$ has units of measure equal to $\left[\mathrm{mol} \mathrm{m}^{-3} \mathrm{~s}^{-1}\right]$. To couple the solute in the fracture with the one in the surrounding porous media, we consider the following interface condition

$$
\epsilon_{\gamma}\left(\operatorname{tr} \chi_{\Omega} \cdot \boldsymbol{n}_{\gamma}-\operatorname{tr} \boldsymbol{q}_{\Omega} \cdot \boldsymbol{n}_{\gamma} \operatorname{tr} u_{\Omega}\right)+\delta_{\gamma}\left(u_{\gamma}-\operatorname{tr} u_{\Omega}\right)=0 \quad \text { on } \gamma \times(0, T) .
$$

For the precipitate in the fracture $w_{\gamma}$, being the original model an ordinary differential equation valid for each point in $\Omega$ the reduced model becomes simply

$$
\partial_{t}\left(\epsilon_{\gamma} w_{\gamma}\right)-\epsilon_{\gamma} r_{w}\left(u_{\gamma}, w_{\gamma} ; \theta_{\gamma}\right)=0 \quad \text { in } \gamma \times(0, T) .
$$

The boundary and initial conditions for the reduced solute and precipitate are finally given by

$$
\begin{array}{ll}
\operatorname{tr} \boldsymbol{\chi}_{\gamma} \cdot \boldsymbol{n}_{\partial \gamma}=\chi_{\partial \gamma} & \text { on } \partial_{e} \gamma \times(0, T) \\
\operatorname{tr} u_{\gamma}=u_{\partial \gamma} & \text { on } \partial_{n} \gamma \times(0, T) \\
\operatorname{tr} \boldsymbol{\chi}_{\gamma} \cdot \boldsymbol{n}_{\partial \gamma}=0 & \text { on } \partial_{i} \gamma \times(0, T), \\
u_{\gamma}(t=0)=u_{\gamma, 0} & \text { in } \gamma \times\{0\} \\
w_{\gamma}(t=0)=w_{\gamma, 0} & \text { in } \gamma \times\{0\}
\end{array}
$$


where $\chi_{\partial \gamma}$ in $\left[\mathrm{mol} \mathrm{m}^{-1} \mathrm{~s}^{-1}\right]$ and $u_{\partial \gamma}$ in $\left[\mathrm{mol} \mathrm{m}^{-3}\right]$ are the given boundary conditions for the flux and solute, respectively. The third conditions is valid for the internal tips, while $u_{\gamma, 0}$ in $\left[\mathrm{mol} \mathrm{m}^{-3}\right]$ and $w_{\gamma, 0}$ in $\left[\mathrm{mol} \mathrm{m}^{-3}\right]$ are the initial conditions in the fracture for the solute and precipitate, respectively. System (12) describes the evolution of the solute and precipitate in the fracture.

\subsection{Permeability and aperture model}

We assume that both components of $k$ follow a cubic law which relates them to the aperture, more precisely

$$
k_{\gamma}\left(\epsilon_{\gamma}\right)=k_{\gamma, 0} \frac{\epsilon_{\gamma}^{2}}{\epsilon_{\gamma, 0}^{2}} \quad \text { and } \quad \kappa_{\gamma}\left(\epsilon_{\gamma}\right)=\kappa_{\gamma, 0} \frac{\epsilon_{\gamma}^{2}}{\epsilon_{\gamma, 0}^{2}},
$$

where $k_{\gamma, 0}\left[\mathrm{~m}^{2}\right]$ and $\kappa_{\gamma, 0}\left[\mathrm{~m}^{2}\right]$ are reference coefficients along and across the fracture, respectively, and $\epsilon_{\gamma, 0}>0$ in [m] is the initial aperture. Finally, we consider a similar law of (5) to describe the evolution of the fracture aperture $\epsilon_{\gamma}$. We have

$$
\begin{array}{ll}
\partial_{t} \epsilon_{\gamma}+\eta_{\gamma} \epsilon_{\gamma} \partial_{t} w_{\gamma}=0 & \text { in } \gamma \times(0, T), \\
\epsilon_{\gamma}(t=0)=\epsilon_{\gamma, 0} & \text { in } \gamma \times\{0\}
\end{array},
$$

here $\eta_{\gamma}$ in $\left[\mathrm{m}^{3} \mathrm{~mol}^{-1}\right]$ represents the rate of deposition of the solute at the fracture walls.

\subsection{Complete reduced model}

The complete setting for a fractured porous media considers the model presented in Subsection 2.5 for the surrounding porous media $\Omega$. To describe the evolution in $\gamma$ of the flow, heat, precipitate and solute as well as the permeability and fracture aperture variations the model is coupled with (10), 111), (12), (13), and (14). Also in this case the system is fully coupled, non-linear and possibly non-smooth.

\subsection{Multiple fracture intersections}

The previously described models extend straightforwardly to the case of multiple fractures that do not intersect to each other. In this case equations $110,, 11],(12),(13)$, and $(14)$ are written for each fracture which, separately, are coupled with the porous media. A different case is when two or more fractures intersect each other and a flow exchange between them can take place. Moreover, due to specific physical properties in the vicinity of the intersection it is a common approach to allow for different data at the intersection, see [32, 41, 37, 18, 30] to name a few.

In this work we consider this approach applied to each equation of the model. We denote an intersection with $\iota$, which can be a line if $n=3$ or a point if $n=2$. Refer to Figure 4 . If the intersection is mono-dimensional then it can be seen as a channel where fluid flow can occur, it is thus approximated with a reduced model similar to $10,,(11),(12),(13)$, and (14) where the aperture now represents the cross sectional area of the intersection. If the intersection is zero-dimensional, resulting also from multiple-intersections of two-dimensional fractures, the treatment is the following.

As did before, we indicate with $\boldsymbol{n}_{\partial \gamma_{i}}$ the normal pointing outward from $\gamma_{i}$ towards the intersection and such that it is also tangent to the fracture $\gamma_{i}$. At the intersection we impose the following conditions for the flow problem

$$
\begin{array}{ll}
\mu \epsilon_{\iota} \operatorname{tr} \boldsymbol{q}_{\gamma_{i}} \cdot \boldsymbol{n}_{\partial \gamma_{i}}+\kappa_{\gamma_{i}}\left(\epsilon_{\iota}\right)\left(p_{\iota}-\operatorname{tr} p_{\gamma_{i}}\right)=0 & \text { on } \iota \times(0, T) . \\
\partial_{t} \epsilon_{\iota}+\nabla_{\iota} \cdot \boldsymbol{q}=0 &
\end{array}
$$




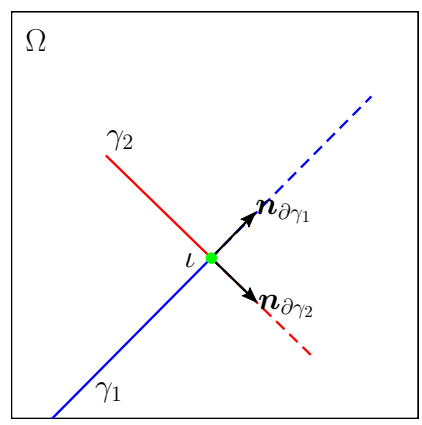

Figure 4: Example of two intersecting fractures with some nomenclature considered.

where $\nabla_{\iota}$. is the zero dimensional conservation operator, defined as

$$
\nabla_{\iota} \cdot \boldsymbol{\nu}=-\sum_{i \in N_{\iota}} \operatorname{tr} \boldsymbol{\nu}_{\gamma_{i}} \cdot \boldsymbol{n}_{\partial \gamma_{i}}
$$

with $N_{\iota}$ the set of fractures meeting in $\iota$ and $\boldsymbol{\nu}=\left(\boldsymbol{\nu}_{\Omega}, \boldsymbol{\nu}_{\gamma_{1}}, \ldots, \boldsymbol{\nu}_{\gamma_{N}}\right)$ the extended compound with $N$ fractures in the problem. In the previous equation (4.7) $p_{\iota}$ is the pressure at the intersection and $\kappa_{\gamma_{i}}$ a fracture specific permeability associated to $\iota$ which can depend on $\epsilon_{\iota}$. Where the latter is the measure of the intersection in the original equi-dimensional framework.

For the heat equation the coupling conditions are given by

$$
\begin{array}{ll}
\epsilon_{\iota}\left(\operatorname{tr} \boldsymbol{\tau}_{\gamma_{i}} \cdot \boldsymbol{n}_{\partial \gamma_{i}}-\rho_{w} c_{w} \operatorname{tr} \boldsymbol{q}_{\gamma_{i}} \cdot \boldsymbol{n}_{\partial \gamma_{i}} \operatorname{tr} \theta_{\gamma_{i}}\right)+\lambda_{w}\left(\theta_{\iota}-\operatorname{tr} \theta_{\gamma_{i}}\right)=0 & \text { on } \iota \times(0, T), \\
\rho_{w} c_{w} \partial_{t}\left(\epsilon_{\iota} \theta_{\iota}\right)+\nabla_{\iota} \cdot \boldsymbol{\tau}=0 &
\end{array}
$$

where in this case $\theta_{\iota}$ is the temperature associated to the intersection $\iota$. For the solute equations the coupling conditions in $\iota$ are given by

$$
\begin{aligned}
& \epsilon_{\iota}\left(\operatorname{tr} \boldsymbol{\chi}_{\gamma_{i}} \cdot \boldsymbol{n}_{\partial \gamma_{i}}-\operatorname{tr} \boldsymbol{q}_{\gamma_{i}} \cdot \boldsymbol{n}_{\partial \gamma_{i}} \operatorname{tr} u_{\gamma_{i}}\right)+\delta_{\iota}\left(u_{\iota}-\operatorname{tr} u_{\gamma_{i}}\right)=0 \\
& \partial_{t}\left(\epsilon_{\iota} u_{\iota}\right)+\nabla_{\iota} \cdot \boldsymbol{\chi}+\epsilon_{\iota} r_{w}\left(u_{\iota}, w_{\iota} ; \theta_{\iota}\right)=0
\end{aligned}
$$

with $u_{\iota}$ and $w_{\iota}$ the solute and precipitate in the intersection, while for the precipitate we have

$$
\partial_{t}\left(\epsilon_{\iota} w_{\iota}\right)-\epsilon_{\iota} r_{w}\left(u_{\iota}, w_{\iota} ; \theta_{\iota}\right)=0 \quad \text { on } \iota \times(0, T) .
$$

Finally, we can extend also the model for the parameter $\epsilon_{\iota}$ as well as for the permeability associated to the intersections. We have the following

$$
\kappa_{\gamma_{i}}\left(\epsilon_{\iota}\right)=\kappa_{\gamma_{i}, 0} \frac{\epsilon_{\iota}^{2}}{\epsilon_{\iota, 0}^{2}} \quad \text { and } \quad \begin{array}{ll}
\partial_{t} \epsilon_{\iota}+\eta_{\iota} \epsilon_{\iota} \partial_{t} w_{\iota}=0 & \text { in } \iota \times(0, T), \\
\epsilon_{\iota}(t=0)=\epsilon_{\iota, 0} & \text { in } \iota \times\{0\}
\end{array},
$$

where $\kappa_{\gamma_{i}, 0}$ and $\epsilon_{\iota, 0}$ are initial values of $\kappa_{\gamma_{i}}$ and $\epsilon_{\iota}$, respectively.

\section{Discretization}

As discussed in Subsection 4.6, the problem is fully coupled. We adopt here a (first order in time) splitting scheme such that legacy codes can be used for its numerical solution. Due to 
the relation (8), it is common to solve the reaction step with an explicit scheme and split the diffusion and advection parts of (3a) and $12 \mathrm{a})$.

The rock domain $\Omega$ is approximated by a grid $\Omega_{h}$ of non-overlapping elements, whose regularity is related to the chosen spatial numerical scheme, that completely cover $\Omega$ itself. We consider here a conforming [52, 55] approximation of the fracture grids with respect to the surrounding porous media grid, meaning that fracture cells are geometrically identical to faces (or edges in 2D) of the porous media grid. We indicate a generic fracture grid as $\gamma_{h}$. The time interval $(0, T)$ is divided, for simplicity, in equally spaced time steps $\Delta t$ such that $N \Delta t=T$, with $N$ the number of time steps. Finally, we indicate with $t^{n}=n \Delta t$ and with a super-script $n$, or $n+1$, the value of variables or data computed at time $t^{n}$, or $t^{n+1}$.

\subsection{The temporal splitting scheme}

In this part we introduce the splitting scheme to solve the global problem introduced in Subsection 4.6. Variables and data are considered semi-discretized in time but not yet in space.

Scheme 1 (Temporal splitting scheme). We set the initial condition as

$$
\begin{gathered}
\phi_{\Omega}^{0}=\phi_{\Omega, 0} \quad \epsilon_{\gamma}^{0}=\epsilon_{\gamma, 0} \quad \theta_{\Omega}^{0}=\theta_{\Omega, 0} \quad \theta_{\gamma}^{0}=\theta_{\gamma, 0} \quad u_{\Omega}^{0}=u_{\Omega, 0} \\
u_{\gamma}^{0}=u_{\gamma, 0} \quad w_{\Omega}^{-1}=w_{\Omega}^{0}=w_{\Omega, 0} \quad w_{\gamma}^{-1}=w_{\gamma}^{0}=w_{\gamma, 0} .
\end{gathered}
$$

In each time step from $t^{n}$ to $t^{n+1}$ we perform the following steps:

1. Extrapolate the precipitate concentration to get a better estimate of porosity, see [44, 1], obtaining

$$
w_{\Omega}^{*}=2 w_{\Omega}^{n}-w_{\Omega}^{n-1} \quad \text { and } \quad w_{\gamma}^{*}=2 w_{\gamma}^{n}-w_{\gamma}^{n-1} .
$$

2. Compute the corresponding porosity and aperture with an implicit discretization of (5) and (14), respectively to get

$$
\phi_{\Omega}^{*}=\frac{\phi_{\Omega}^{n}}{1+\eta_{\Omega}\left(w_{\Omega}^{*}-w_{\Omega}^{n}\right)} \quad \text { and } \quad \epsilon_{\gamma}^{*}=\frac{\epsilon_{\gamma}^{n}}{1+\eta_{\gamma}\left(w_{\gamma}^{*}-w_{\gamma}^{n}\right)} .
$$

Note that, with this approximation, these extrapolated values of porosity and aperture cannot become negative when the precipitate increases.

3. Update porous media permeability $k_{\Omega}\left(\phi_{\Omega}^{*}\right)$ and fracture normal and tangential permeabilities $k_{\gamma}\left(\epsilon_{\gamma}^{*}\right)$ and $\kappa_{\gamma}\left(\epsilon_{\gamma}^{*}\right)$ according to (4) and (13), respectively.

4. With $\left(\phi_{\Omega}^{*}, \epsilon_{\gamma}^{*}\right)$ and the computed permeabilities, solve the Darcy problem, (1) and (10), to get $\left(\boldsymbol{q}_{\Omega}^{n+1}, p_{\Omega}^{n+1}\right)$ and $\left(\boldsymbol{q}_{\gamma}^{n+1}, p_{\gamma}^{n+1}\right)$, respectively.

5. With the advective fields $\left(\boldsymbol{q}_{\Omega}^{n+1}, \boldsymbol{q}_{\gamma}^{n+1}\right)$ computed in the previous point, solve the heat equations (2) and (11) to obtain temperature distribution $\theta_{\Omega}^{n+1}$ and $\theta_{\gamma}^{n+1}$, respectively.

6. With the advective fields $\left(\boldsymbol{q}_{\Omega}^{n+1}, \boldsymbol{q}_{\gamma}^{n+1}\right)$, solve the advection-diffusion parts for the solute (3) and $(12)$ to get the intermediate $u_{\Omega}^{n+\frac{1}{2}}$ and $u_{\gamma}^{n+\frac{1}{2}}$, respectively. 
7. Since in the previous advection-diffusion step we have accounted for porosity changes using $\phi_{\Omega}^{*}$, the new concentration $u_{\Omega}^{n+\frac{1}{2}}$ and $u_{\gamma}^{n+\frac{1}{2}}$ accounts for the change in pore volume, thus, the precipitate needs to be updated accordingly computing

$$
w_{\Omega}^{n+\frac{1}{2}}=w_{\Omega}^{n} \frac{\phi_{\Omega}^{n}}{\phi_{\Omega}^{*}} \quad \text { and } \quad w_{\gamma}^{n+\frac{1}{2}}=w_{\gamma}^{n} \frac{\epsilon_{\gamma}^{n}}{\epsilon_{\gamma}^{*}} .
$$

8. Solve the reaction step starting from $\left(w_{\Omega}^{n+\frac{1}{2}}, w_{\gamma}^{n+\frac{1}{2}}\right)$ and $\left(u_{\Omega}^{n+\frac{1}{2}}, u_{\gamma}^{n+\frac{1}{2}}\right)$ to get $\left(w_{\Omega}^{* *}, w_{\gamma}^{* *}\right)$ and $\left(u_{\Omega}^{* *}, u_{\gamma}^{* *}\right)$.

9. At this point we can update the porosity and aperture with the true concentration of precipitate at time $n+1$, that is

$$
\phi_{\Omega}^{n+1}=\frac{\phi_{\Omega}^{n}}{1+\eta_{\Omega}\left(w_{\Omega}^{* *}-w_{\Omega}^{n}\right)} \quad \text { and } \quad \epsilon_{\gamma}^{n+1}=\frac{\epsilon_{\gamma}^{n}}{1+\eta_{\gamma}\left(w_{\gamma}^{* *}-w_{\gamma}^{n}\right)} .
$$

10. Finally, we correct the concentrations to account for the difference between the extrapolated and "true" new porosity and aperture, namely $\phi_{\Omega}^{*}$ and $\phi_{\Omega}^{n+1}$ and $\epsilon_{\gamma}^{*}$ and $\epsilon_{\gamma}^{n+1}$, respectively. To ensure mass conservation, we thus compute

$$
w_{\Omega}^{n+1}=w_{\Omega}^{* *} \frac{\phi_{\Omega}^{*}}{\phi_{\Omega}^{n+1}} \quad u_{\Omega}^{n+1}=u_{\Omega}^{* *} \frac{\phi_{\Omega}^{*}}{\phi_{\Omega}^{n+1}} \quad w_{\gamma}^{n+1}=w_{\gamma}^{* *} \frac{\epsilon_{\gamma}^{*}}{\epsilon_{\gamma}^{n+1}} \quad u_{\gamma}^{n+1}=u_{\gamma}^{* *} \frac{\epsilon_{\gamma}^{*}}{\epsilon_{\gamma}^{n+1}} .
$$

In the following parts we detail the temporal solution of some of the previous points. In section 5.1.1 we discuss Step 4 in section 5.1.2 the discretized heat model from Step 5 in section 5.1 .3 the Step 6, finally in Subsection 5.2 the reaction part in Step 8 .

Remark 1. Since the order of convergence in time is bounded by the chosen splitting scheme, thus of order one, we generally consider low order schemes when high accuracy is not needed.

Remark 2. Steps 5 and 6 can be solve in parallel increasing the performances of the code. Moreover, the computation of the porosity, aperture, and reaction parts are done cell by cell and they are thus embarrassingly parallel.

\subsubsection{Temporal discretization of Darcy model}

The Implicit Euler (IE) scheme is used to discretize the temporal derivative of the porosity, with $\phi_{\Omega}^{*}$ from Step 2 and $\phi_{\Omega}^{n}$ from the previous time step. In 1a), the bulk part of Step 4 , i.e. the Darcy problem in $\Omega$ is thus solved as

$$
\begin{array}{ll}
\mu \boldsymbol{q}_{\Omega}^{n+1}+k\left(\phi_{\Omega}^{*}\right) \nabla p_{\Omega}^{n+1}=\mathbf{0} & \text { in } \Omega \times\left(t^{n}, t^{n+1}\right) \\
\phi_{\Omega}^{*}-\phi_{\Omega}^{n}+\Delta t \nabla_{\Omega} \cdot \boldsymbol{q}^{n+1}+\Delta t f_{\Omega}^{n+1}=0 &
\end{array}
$$

The term involving the porosity is now a given data and can be considered as additional source term. Boundary conditions $(1 \mathrm{~b})$ are adapted accordingly.

For the solution in fracture of Step 4 we proceed similarly, the aperture time derivative is dicretized with IE by considering $\epsilon_{\gamma}^{*}$ and $\epsilon_{\gamma}^{n}$ from the previous time step. From 10a the scheme becomes

$$
\begin{aligned}
& \mu \boldsymbol{q}_{\gamma}^{n+1}+\epsilon_{\gamma}^{*} k_{\gamma}\left(\epsilon_{\gamma}^{*}\right) \nabla p_{\gamma}^{n+1}=\mathbf{0} \\
& \epsilon_{\gamma}^{*}-\epsilon_{\gamma}^{n}+\Delta t \nabla_{\gamma} \cdot \boldsymbol{q}^{n+1}+\Delta t \epsilon_{\gamma}^{*} f_{\gamma}^{n+1}=0
\end{aligned} \quad \text { in } \gamma \times\left(t^{n}, t^{n+1}\right)
$$


Also in this case the boundary conditions $10 \mathrm{c}$ are discretized likewise.

The discretization of the coupling term (10b), between the fracture and the bulk for the flow model, uses the extrapolated value of the aperture

$$
\mu \epsilon_{\gamma}^{*} \operatorname{tr} \boldsymbol{q}_{\Omega}^{n+1} \cdot \boldsymbol{n}_{\gamma}+\kappa_{\gamma}\left(\epsilon_{\gamma}^{*}\right)\left(p_{\gamma}^{n+1}-\operatorname{tr} p_{\Omega}^{n+1}\right)=0 \quad \text { on } \gamma \times\left(t^{n}, t^{n+1}\right) .
$$

Because problems in the fractures and surrounding porous media are coupled, problem 15 is solved to obtain the final value of $\left(\boldsymbol{q}_{\Omega}^{n+1}, p_{\Omega}^{n+1}\right)$ and $\left(\boldsymbol{q}_{\gamma}^{n+1}, p_{\gamma}^{n+1}\right)$.

\subsubsection{Temporal discretization of heat model}

By considering the temperature at previous time step $\theta_{\Omega}^{n}$ and $\theta_{\gamma}^{n}$, also the heat equation is discretized in Step 5 with the IE scheme. We obtain the following expression for the heat problem 2a) in $\Omega$

$$
\begin{aligned}
& \boldsymbol{\tau}_{\Omega}^{n+1}-\rho_{w} c_{w} \boldsymbol{q}_{\Omega}^{n+1} \theta_{\Omega}^{n+1}+\lambda\left(\phi_{\Omega}^{*}\right) \nabla \theta_{\Omega}^{n+1}=\mathbf{0} \\
& c\left(\phi_{\Omega}^{*}\right) \theta_{\Omega}^{n+1}-c\left(\phi_{\Omega}^{n}\right) \theta_{\Omega}^{n}+\Delta t \nabla_{\Omega} \cdot \boldsymbol{\tau}^{n+1}+\Delta t j_{\Omega}^{n+1}=0
\end{aligned} \quad \text { in } \Omega \times\left(t^{n}, t^{n+1}\right),
$$

where $c\left(\phi_{\Omega}^{*}\right)$ and $\lambda\left(\phi_{\Omega}^{*}\right)$ are the extrapolated values of the effective thermal capacity and conductivity at time $n+1$ computed using the value of $\phi_{\Omega}^{*}$ in $(2 \mathrm{~b})$ and $(2 \mathrm{c})$ as

$$
c\left(\phi_{\Omega}^{*}\right)=\phi_{\Omega}^{*} \rho_{w} c_{w}+\left(1-\phi_{\Omega}^{*}\right) \rho_{s} c_{s} \quad \text { and } \quad \lambda\left(\phi_{\Omega}^{*}\right)=\lambda_{w}^{\phi_{\Omega}^{*}} \lambda_{s}^{1-\phi_{\Omega}^{*}} .
$$

For the fracture part of Step 5 , the time derivative discretized with IE considers the extrapolated value of aperture $\epsilon_{\gamma}^{*}$. We obtain the discretized version of (11a) given by

$$
\begin{aligned}
& \boldsymbol{\tau}_{\gamma}^{n+1}-\rho_{w} c_{w} \boldsymbol{q}_{\gamma}^{n+1} \theta_{\gamma}^{n+1}+\epsilon_{\gamma}^{*} \lambda_{w} \nabla \theta_{\gamma}^{n+1}=\mathbf{0} \\
& \rho_{w} c_{w}\left(\epsilon_{\gamma}^{*} \theta_{\gamma}^{n+1}-\epsilon_{\gamma}^{n} \theta_{\gamma}^{n}\right)+\Delta t \nabla_{\gamma} \cdot \boldsymbol{\tau}^{n+1}+\Delta t j_{\gamma}^{n+1}=0
\end{aligned} \quad \text { in } \gamma \times\left(t^{n}, t^{n+1}\right)
$$

The discretization of boundary conditions $(11 \mathrm{c})$ follows immediately.

The temporal discretization of the interface condition $111 \mathrm{~b}$ between the fracture and surrounding porous media in Step 5 is the following

$$
\epsilon_{\gamma}^{*}\left(\operatorname{tr} \boldsymbol{\tau}_{\Omega}^{n+1} \cdot \boldsymbol{n}_{\gamma}-\rho_{w} c_{w} \operatorname{tr} \boldsymbol{q}_{\Omega}^{n+1} \cdot \boldsymbol{n}_{\gamma} \operatorname{tr} \theta_{\Omega}^{n+1}\right)+\lambda_{w}\left(\theta_{\gamma}^{n+1}-\operatorname{tr} \theta_{\Omega}^{n+1}\right)=0 \quad \text { on } \gamma \times\left(t^{n}, t^{n+1}\right) .
$$

The coupled problem 16 is solved to obtain the final value of the primary variables $\theta_{\Omega}^{n+1}$ and $\theta_{\gamma}^{n+1}$.

\subsubsection{Temporal discretization of advection-diffusion solute model}

The temporal discretization of the advection and diffusion parts of the solute in Step 6 is obtained by setting the corresponding reaction term to zero. We consider the IE scheme for the temporal discretization of $(3 \mathrm{a})$, which, in the porous matrix, reads

$$
\begin{array}{ll}
\chi_{\Omega}^{n+1}-\boldsymbol{q}_{\Omega}^{n+1} u^{n+1}+\phi_{\Omega}^{*} d \nabla u_{\Omega}^{n+1}=\mathbf{0} & \text { in } \Omega \times\left(t^{n}, t^{n+1}\right), \\
\phi_{\Omega}^{*} u_{\Omega}^{n+1}-\phi_{\Omega}^{n} u_{\Omega}^{n}+\Delta t \nabla_{\Omega} \cdot \chi^{n+1}=0 &
\end{array}
$$

with boundary conditions of the solute in $\Omega(3 \mathrm{c})$ easily implemented.

The fracture part of Step 6 consists in solving equation $12 \mathrm{a}$ with null reaction term. Also in this case we take extrapolated aperture $\epsilon_{\gamma}^{*}$ in the time discretization. The equations become

$$
\begin{aligned}
& \boldsymbol{\chi}_{\gamma}^{n+1}-\boldsymbol{q}_{\gamma}^{n+1} u_{\gamma}^{n+1}+\epsilon_{\gamma}^{*} d_{\gamma} \nabla u_{\gamma}^{n+1}=\mathbf{0} \\
& \epsilon_{\gamma}^{*} u_{\gamma}^{n+1}-\epsilon_{\gamma}^{n} u_{\gamma}^{n}+\Delta t \nabla_{\gamma} \cdot \boldsymbol{\chi}^{n+1}=0
\end{aligned}
$$$$
\text { in } \gamma \times\left(t^{n}, t^{n+1}\right) \text {. }
$$ 


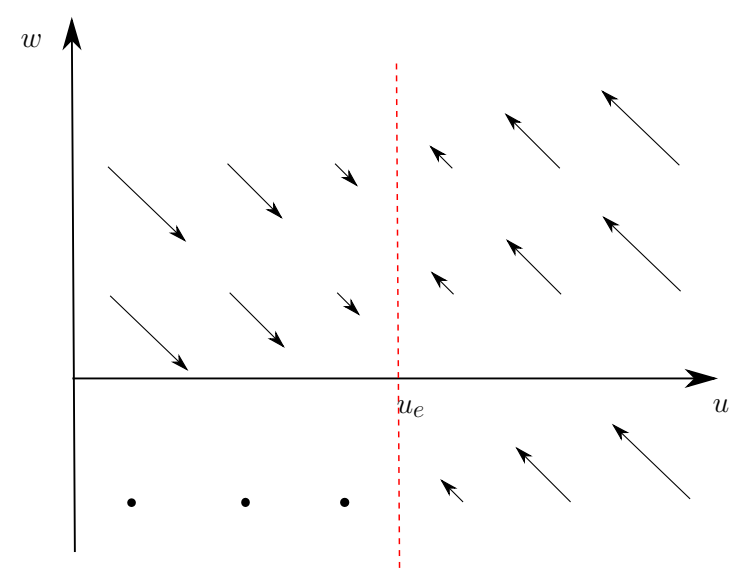

Figure 5: Qualitative representation of the vector forcing term $\boldsymbol{r}_{w}$ in the phase space $(u, w)$.

Finally, the coupling conditions $12 \mathrm{~b}$ between the fracture and surrounding porous media are discretized as

$$
\epsilon_{\gamma}^{*}\left(\operatorname{tr} \chi_{\Omega}^{n+1} \cdot \boldsymbol{n}_{\gamma}-\operatorname{tr} \boldsymbol{q}_{\Omega}^{n+1} \cdot \boldsymbol{n}_{\gamma} \operatorname{tr} u_{\Omega}^{n+1}\right)+\delta\left(u_{\gamma}^{n+1}-\operatorname{tr} u_{\Omega}^{n+1}\right)=0 \quad \text { on } \gamma \times\left(t^{n}, t^{n+1}\right) .
$$
$u_{\gamma}^{n+1}$.

Coupled problem (17) is solved to obtain the final value of the primary variables $u_{\Omega}^{n+1}$ and

\subsection{Integration in time of the discontinuous reaction problem}

As explained in the previous section we rely on a first order splitting to the solute equation in the bulk and in the fractures to separate the advection and diffusion part from the reaction term. This is motivated by the discontinuous nature of the reaction term, which benefits from an ad hoc numerical treatment. Starting from the intermediate solution $\left(u_{\Omega}^{n+\frac{1}{2}}, w_{\Omega}^{n+\frac{1}{2}}\right),\left(u_{\gamma}^{n+\frac{1}{2}}, w_{\gamma}^{n+\frac{1}{2}}\right)$ we integrate, for each degree of freedom in the porous medium and in the fractures, a system of two ordinary differential equations. Note that, indeed, after discretization in space, $u$ and $w$ will be approximated as piecewise constant, thus, with an abuse of notation, we write for each cell the following system of ODEs

$$
d_{t}[u, w]^{\top} \in \boldsymbol{r}_{w}(u, v ; \theta) \quad \text { with } \quad \boldsymbol{r}_{w}(u, w ; \theta)=\left[-r_{w}(u, w ; \theta), r_{w}(u, w ; \theta)\right]^{\top},
$$

where $r_{w}$ is defined as in (8). Note that $(18)$ is written as a differential inclusion because, strictly speaking, the forcing term is not defined at $w=0$. It is also important to highlight that the discontinuity depends on the solution itself and not simply on time. The vector forcing term $\boldsymbol{r}_{w}$ is represented qualitatively in Figure 5 where we can observe that it is discontinuous across the line $w=0$ for $u<u_{e}$, in other words, when the solute concentration is such that we should have precipitate dissolution. We can define

$$
\boldsymbol{r}_{w}(u, w ; \theta)=\left\{\begin{array}{ll}
\boldsymbol{r}_{w}^{+}(u ; \theta) & \text { if } w>0 \\
\boldsymbol{r}_{w}^{-}(u ; \theta) & \text { if } w<0
\end{array},\right.
$$

where $\boldsymbol{r}_{w}^{+}$and $\boldsymbol{r}_{w}^{-}$are defined as

$$
\begin{gathered}
\boldsymbol{r}_{w}^{+}(u ; \theta)=\lambda^{-}(\theta)[-(r(u)-1),(r(u)-1)]^{\top}, \\
\boldsymbol{r}_{w}^{-}(u ; \theta)=\lambda^{-}(\theta)[-\max (r(u)-1,0), \max (r(u)-1,0)]^{\top} .
\end{gathered}
$$




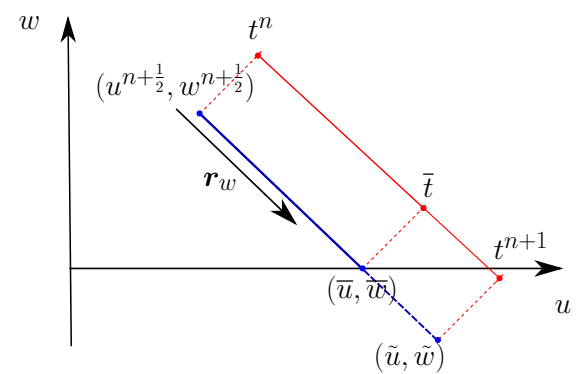

Figure 6: splitting of an integration step across the discontinuity.

Equation (18) is integrated numerically with an explicit scheme (Explicit Euler or two-stages Runge-Kutta) combined with an event location strategy. For the sake of simplicity we will describe the procedure in the case of the EE scheme. In particular at each step we need to:

1. detect if and when the solution reaches the discontinuity threshold, i.e. the line $w=0$. Note that this instant, denoted as $\bar{t}$, usually does not coincide with $t^{n}$ or $t^{n+1}$;

2. at $\bar{t}$ stop and restart the numerical integration with a suitable forcing term: $\boldsymbol{r}_{w}^{+}, \boldsymbol{r}_{w}^{-}$or a convex combination of the two.

\subsubsection{Detection of the event}

Following 24] we perform a tentative integration step starting from $t^{n}$ and the initial conditions $\left(u^{n+\frac{1}{2}}, w^{n+\frac{1}{2}}\right)$ to obtain

$$
\begin{gathered}
\tilde{u}=u^{n+\frac{1}{2}}-\Delta \operatorname{tr}_{w}\left(u^{n+\frac{1}{2}}, w^{n+\frac{1}{2}}\right) \\
\tilde{w}=w^{n+\frac{1}{2}}+\Delta \operatorname{tr}_{w}\left(u^{n+\frac{1}{2}}, w^{n+\frac{1}{2}}\right)
\end{gathered} .
$$

We then check whether $\tilde{w}<0$ : if this is the case it means that in the $n$-th integration step we are crossing the discontinuity threshold. We proceed searching for the exact time of the event by defining $w(\xi)=w^{n+\frac{1}{2}}+\xi \Delta t r_{w}\left(u^{n+\frac{1}{2}}, w^{n+\frac{1}{2}}\right)$ and search for $\bar{\xi}$ such that $w(\bar{\xi})=0$ by means of a suitable iterative method. Once we have detected the time of transition $\bar{t}=t^{n}+\bar{\xi} \Delta t$ we perform the following steps:

1. integrate the equations from $t^{n}$ to $\bar{t}$ with the "old" value of the right hand side obtaining $\bar{u}$ and $\bar{w}$ as shown in Figure 6 .

2. integrate from $t^{n}+\bar{\xi} \Delta t$ to $t^{n+1}$ with a new value of the right hand side.

\subsubsection{Choice of the right hand side}

To determine the behavior of the solution at the discontinuity we let $\boldsymbol{n}$ be the normal to the surface of discontinuity in the phase space, in our case $\boldsymbol{n}=[0,1]^{T}$, and observe the sign of $\boldsymbol{r}_{w}^{ \pm} \cdot \boldsymbol{n}$. 
Suppose for the sake of simplicity that we start from the "+" region, i.e. $w^{n+\frac{1}{2}}>0$ and $u<u_{e}$ : thus $\boldsymbol{r}_{w}^{+} \cdot \boldsymbol{n}<0$ meaning that precipitate is decreasing and we are approaching the discontinuity. On the opposite side, $\boldsymbol{r}_{w}^{-} \cdot \boldsymbol{n}=0$. With these conditions the solution, after $\bar{t}$, should slide on the discontinuity, i.e. we finish the integration of the time step with forcing term $\boldsymbol{r}_{w}^{-}$, which is null until the solute reaches its equilibrium concentration. In other words, stops if the precipitate is not present.

Note that, if $u>u_{e}, \boldsymbol{r}_{w}^{ \pm} \cdot \boldsymbol{n}>0$, and there is no discontinuity across $w=0$ because in this case the net reaction rate yields an increase of precipitate.

\subsection{Spatial discretization}

The discretization in space of the problems in Subsection 4.6 is not the main focus of this work and relies on a standard approach. Since equations are in mixed-dimensions, the numerical schemes considered are applied in different dimensions.

The main request for the discretization of the flow problem $\sqrt{15}$ is to obtain a reliable approximation of Darcy velocity that is locally mass conservative. To solve problem (15) we consider two different schemes depending on the geometrical properties of the grids, the Raviart-Thomas of lowest order, see [57, 58, 52, 17, and the mixed virtual element method of lowest degree, see for example [19, 14, 12, 13, 36, 37. Both schemes handle in an accurate way strong variations of the permeability tensor which is a typical situation in the underground. For the numerical solution of problems (16) and (17) we consider an upstream approximation for the advective part and a two-point flux approximation for the diffusion component, see [26, 47, 29, 25].

For the coupling between the fracture and the porous media, for simplicity we assume a conforming strategy meaning that the element of the fracture grids are composed by faces or edges of the porous media elements neighbouring the fracture. Other choices are possible that relax some of the geometrical constraints posed by this approach, see for example [31, 38.

\section{$6 \quad$ Numerical examples}

In this section we present three numerical examples to show the performances of the previously introduced mathematical models and splitting scheme. In particular, in the examples reported in Subsection 6.1 we validate the good properties of the splitting scheme of Scheme 1 presented in Section 5.1. The next examples, presented in Subsection 6.2 and 6.3. consider the full problem with single and multiple fractures, respectively. In these examples, we present the relevant physical effects the proposed model is able to reproduce by increasing the geometrical complexity. These later examples are developed with the library PorePy, a simulation tool for fractured and deformable porous media written in Python, see [49. The scripts associated are freely accessible.

\section{1 $1 \mathrm{D}$ tests}

Let us consider first a simple one dimensional test case on a domain $\Omega=(0,1)$ without any fracture. The goal is to test the reliability of the algorithm presented in Section 5.1. Solute concentration is set, at the initial time, to $u=2 u_{e}$ in the central part of the domain, while $w=0$ everywhere at $t=0$. The advection/diffusion ratio is about 10 , and $C F L=\frac{q \Delta t}{\Delta x} \simeq 8 \cdot 10^{-2}$. The reaction rate is such that $\mathbb{D} a=0.2$, and the coefficient in 13$) \eta_{\Omega}=1$. A pressure drop imposed at the boundaries results in a Darcy velocity $q \simeq 10^{-8}$, however, as a result of porosity changes this value will be perturbed. In Figure 7 we can observe the evolution of $u, w, \phi, q$ and the corresponding mass balance. We observe that $u$ decreases due to precipitation, and is advected towards the right boundary. The precipitate grows, initially, but is later "washed away" by water 

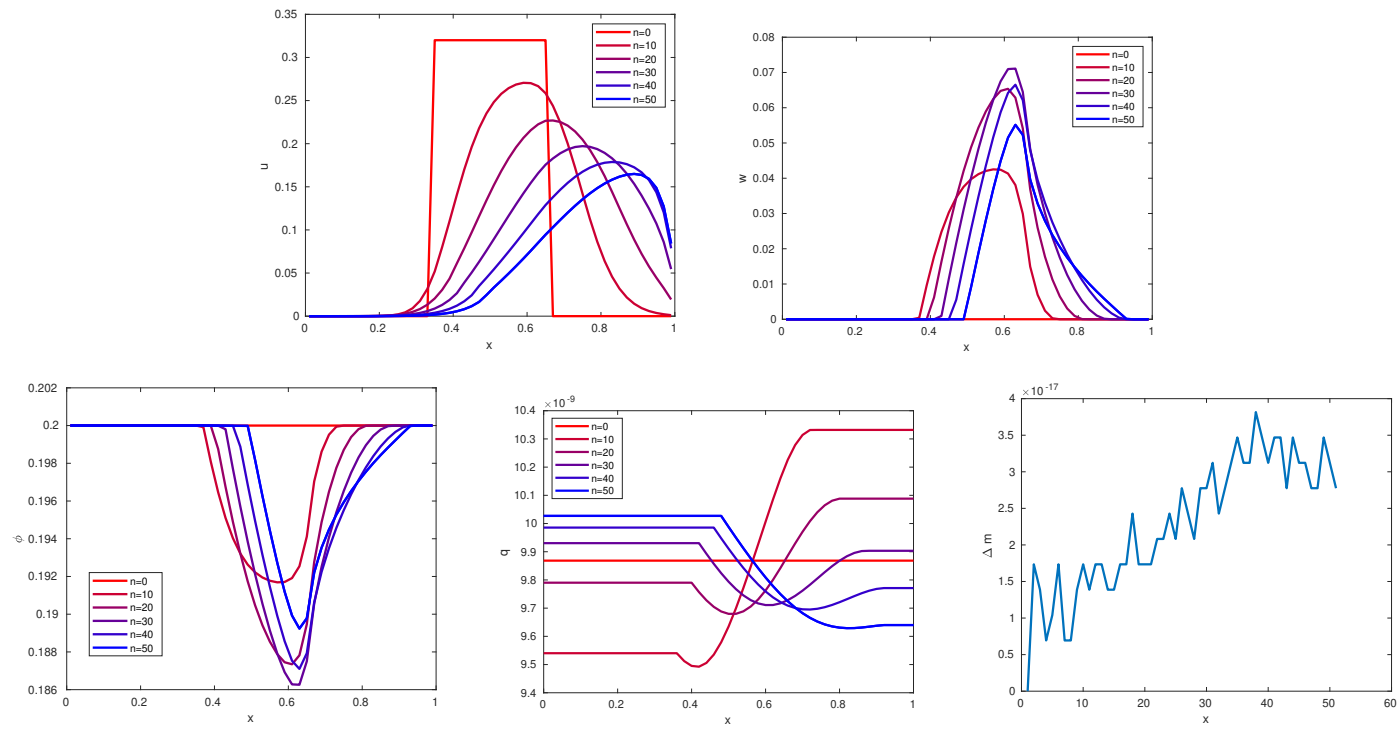

Figure 7: Precipitation and dissolution with a given advection field in 1D at different time steps. Top row: solute e precipitate concentrations. Bottom: porosity distribution, Darcy velocity and mass balance over time.

\begin{tabular}{|c|c|c|c|c|}
\hline$N_{t} \backslash D a(C F L)$ & $0.1\left(8.64 \cdot 10^{-1}\right)$ & $1\left(8.64 \cdot 10^{-2}\right)$ & $10\left(8.64 \cdot 10^{-3}\right)$ & $100\left(8.64 \cdot 10^{-4}\right)$ \\
\hline 50 & $1.4 \cdot 10^{-3}$ & $3.2 \cdot 10^{-3}$ & $1.4 \cdot 10^{-3}$ & $4.9759 \cdot 10^{-4}$ \\
\hline 100 & $1.1 \cdot 10^{-3}$ & $1.8 \cdot 10^{-3}$ & $7.4362 \cdot 10^{-4}$ & $2.7832 \cdot 10^{-4}$ \\
\hline 200 & $7.6036 \cdot 10^{-4}$ & $1.0 \cdot 10^{-3}$ & $3.7707 \cdot 10^{-4}$ & $1.4577 \cdot 10^{-4}$ \\
\hline
\end{tabular}

Table 1: $L^{\infty}$ norm of the difference between the solutions obtained with and without the splitting at the final time. We consider different advection velocities and increase the number of time steps $N_{t}$.

with a solute concentration that is lower than the equilibrium one. The evolution of porosity reflects that of $w$. The quantity $\Delta m$ is computed as the mass loss/gain that is the difference between mass of $u$ and $w$ inside the domain, the outflow of $u$ and the initial mass: we observe that mass is conserved within machine precision.

We use a similar, but simplified setup to evaluate the operator splitting error by comparing the solution obtained with a monolithic method with the one obtained with the first order splitting for advection-diffusion and reaction. In this case we neglect the changes in porosity, consider a constant, given Darcy velocity and take a linear reaction rate $r(u)=u$. The value of Darcy velocity changes at fixed reaction rate to obtain different Damkhöler numbers. We can observe in table 1 that $i)$ the splitting error decreases linearly with $\Delta t$ for $C F L<1$, ii) however, for $C F L$ close to 1 the splitting error does not decrease with the correct rate, and iii) the largest errors are obtained for $\mathbb{D} a=1$, i.e. when advection and reaction occur at the same speed.

Finally, we want to show the impact of Damkhöler number on the precipitate distribution in the presence of a point source to mimic the effect of a fracture in $2 \mathrm{D} / 3 \mathrm{D}$ and predict whether the effect of fracture flow will result in a local or more diffused change in the porous matrix properties. To this aim we start from clean water $(u=0)$ and inject a prescribed concentration $u=2 u_{e}$ at $x=0.5$. The velocity field is given, $q=\bar{q} \operatorname{sign}(x-0.5)$. 
$D a=0.006$

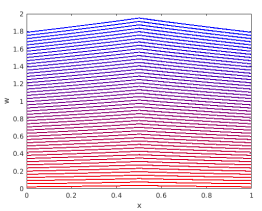

$D a=0.066$

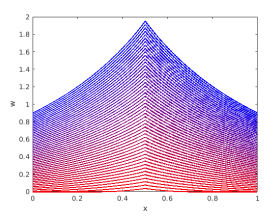

$D a=0.662$

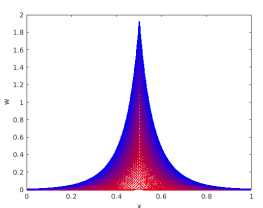

$D a=6.62$

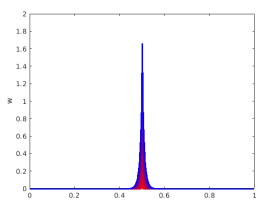

Figure 8: Precipitate concentration for different Darcy velocities, corresponding to a point-wise injection of water with $u=2 u_{e}$ at $x=0.5$.
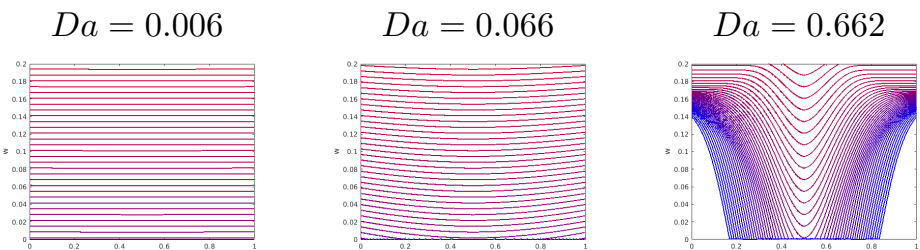

$D a=6.62$

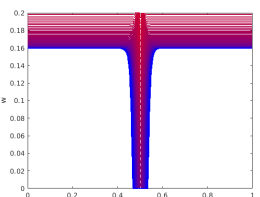

Figure 9: Precipitate concentration for different Darcy velocities, corresponding to a point-wise injection of clean water in a porous matrix with $w=2 u_{e}$ at $x=0.5$.

If we consider different characteristic Darcy velocities or, in other words, different Damkhöhler numbers, we obtain the results represented in Figure 8 , high advection (small $\mathbb{D} a$ ) correspond to a flat solute concentration profile and thus a uniform precipitation, whereas if advection is very slow compared to reaction, precipitation is focused around the injection point.

Conversely, if we inject clean water into the "well" and we start from the initial constant concentrations $u=0, w=2 u_{e}$ we obtain the results in Figure 9, where, for large advection velocities the precipitate is eroded uniformly in the whole domain, whereas for large $\mathbb{D} a$ the effect is concentrated around the injection point.

\subsection{Single fracture}

We consider a single fracture in the unit square domain $\Omega=(0,1)^{2}$. The fracture starts from $(0.1,0)$ and ends at $(0.9,0.8)$, see Figure 10 for a schematic representation of the computational domain. As shown in the figure, we assume that the left and right boundaries are impervious while on the bottom and top we set in-flow and out-flow conditions, respectively. In this example the values of data are artificial and meant to highlight some phenomena, we thus omit units of measure to avoid confusions. We consider two different settings, the common data are defined in Table 2 ,

The porous medium is discretized with approximately 10000 triangles and the fracture with approximately 75 segments. Since the computational grid is made of triangles we can simply employ the lowest order Raviart-Thomas mixed finite element method for the solution of the flow problem.

In the solution plots presented in the sequel, for each time step, we represent on the top left the pressure and Darcy velocity; on the top right the temperature; on the bottom left the solute multiplied by the porosity or aperture; on the bottom centre the precipitate multiplied by the porosity or aperture; and on the bottom right the porosity and aperture. Note that the values, apart from the velocity, are rescaled at each time step to highlight some details of the solution. Moreover, we choose to represent $\phi u, \phi w$ since these quantities, which correspond to the amount 


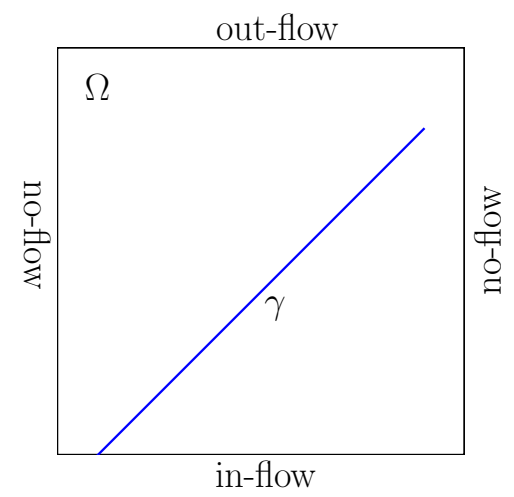

Figure 10: Domain $\Omega$ and fracture $\gamma$ for the example of Subsection 6.2 .

\begin{tabular}{|c|c|c|c|c|}
\hline$\eta_{\Omega}=0.5$ & $\phi_{\Omega, 0}=0.2$ & $\eta_{\gamma}=2$ & $\epsilon_{0}=10^{-2}$ & $k_{0}=1$ \\
\hline$\mu=1$ & $f=0$ & $q_{\partial \Omega}=0$ & $p_{\partial \Omega}^{\text {out-flow }}=0$ & $p_{\partial \Omega}^{\text {in-flow }}=1$ \\
\hline$k_{\gamma, 0}=10^{2}$ & $\kappa_{\gamma, 0}=10^{2}$ & $\kappa_{\gamma, 0}=10^{2}$ & $\mu=1$ & $f_{\gamma}=0$ \\
\hline$q_{\partial \gamma}=0$ & $p_{\partial \gamma}^{\text {out-flow }}=0$ & $p_{\partial \gamma}^{\text {in-flow }}=1$ & $\lambda_{w}=1$ & $\lambda_{s}=10^{-1}$ \\
\hline$\rho_{w} c_{w}=1$ & $\rho_{s} c_{s}=1$ & $j=0$ & $\theta_{0}=1$ & $\tau_{\partial \Omega}=0$ \\
\hline$\theta_{\partial \Omega}^{\text {out }- \text { flow }}=0$ & $\theta_{\partial \Omega}^{\text {in-flow }}=1.5$ & $j_{\gamma}=0$ & $\theta_{\gamma, 0}=1$ & $\tau_{\partial \gamma}=0$ \\
\hline$\theta_{\partial \gamma}^{\text {out-flow }}=0$ & $\theta_{\partial \gamma}^{\text {in-flow }}=1.5$ & $d=1$ & $u_{0}=0$ & $\chi_{\partial \Omega}=0$ \\
\hline$u_{\partial \Omega}^{\text {out-flow }}=0$ & $d_{\gamma}=10^{-1}$ & $\delta_{\gamma}=10^{-1}$ & $u_{\gamma, 0}=0$ & $\chi_{\partial \gamma}=0$ \\
\hline$u_{\partial \gamma}^{\text {out-flow }}=0$ & $\lambda^{-}(\theta)=10 e^{-\frac{4}{\theta}}$ & $r(u)=u^{2}$ & & \\
\hline
\end{tabular}

Table 2: Common data for the examples in Subsection 6.2 and Subsection 6.3 
of solute/precipitate per unit rock volume, are easier to interpret when porosity (and aperture) changes significantly.

\subsubsection{Solute injection}

In this first case we consider the following additional data: the final simulation time is $T=3$ discretized with 60 time steps; $w_{0}=0.3$ and $u_{\partial \Omega}^{\text {inflow }}=2$ for equation (3); $w_{\gamma, 0}=0.3$ and $u_{\partial \gamma}^{\text {inflow }}=2$ for 12 .

In this example, we inject in the porous media from the bottom boundary the solute as well as warm water. As we see in Figure 11, the fracture is highly conductive thus the solute is transported quickly and starts to react first in the fracture. The creation of new precipitate tends to block the fracture making it less and less permeable. The high temperature front accelerates this process making the fracture almost completely clogged, see the plot of fracture aperture at the bottom of Figure 11. Moreover, also the pressure profile exhibits a jump across the fracture and the Darcy velocity drops substantially, both signs of a low fracture permeability. We also see that the precipitate accumulates preferably in the fracture. The porosity of the medium decreases too, first nearby the fracture and after, due to the injection of solute and temperature front, in the whole porous media.

This example, even with simple data, is able to capture interesting phenomena, like the clogging of a high permeable fracture.

\subsubsection{Fracture opening}

In this second case we consider the following additional data: the final simulation time is $T=5$ discretized with 100 time steps; by defining the square $S=\{(x, y) \in \Omega: 0.4 \leq(x, y) \leq 0.6\}$ we have $w_{0}=1$ in $S$ and $w_{0}=0$ elsewhere and $u_{\partial \Omega}^{\text {in-flow }}=0$ for equation (3); $w_{\gamma, 0}=1$ in $\gamma \cap S$ and $w_{\gamma, 0}=0$ otherwise and $u_{\partial \gamma}^{\text {in }- \text { flow }}=0$ for 12 .

In this example, we inject clean water (no solute) in the system which has a block of precipitate in the middle. The precipitate is also contained in the fracture. Since the fracture has high permeability the clean water dissolves first the precipitate in the fracture by forming new solute that is released in the system, as the top of Figure 12 shows. The fracture aperture is thus increased as well as the porosity. After some time, as reported in the bottom of Figure 12 , the high temperature front reaches the block of precipitate also through the porous media. The reaction now becomes faster and dissolves most of the precipitate forming more solute which is transported upward. The pressure profile changes only slightly during the simulation but the Darcy velocity increases visibly in $S$ due to the enhancement of the permeability.

Also in this case, even if the problem setting is simple, we are able to reproduce important phenomena, like the opening of a fracture due to injection of clean water.

\subsection{Multiple fractures}

In this third case we increase the geometrical complexity by considering a problem with 10 intersecting fractures. The geometry of the fractures is taken from the Benchmark 3 of 30 but with different properties. The domain $\Omega=(0,1)^{2}$ and the fractures are shown in Figure 13 For the computational grid, due to the complexity of the fracture network we consider the procedure discussed in [43]: first a Cartesian grid is constructed and then its cells are cut if a fracture is crossing. Since we may obtain cells of arbitrary shape, we adopt the lowest order mixed virtual element method for the discretization of the flow equations. The grid is represented on the right of Figure 13, the fracture network is discretized with approximately 200 segments while the porous media grid consists of approximately 1300 cells. 


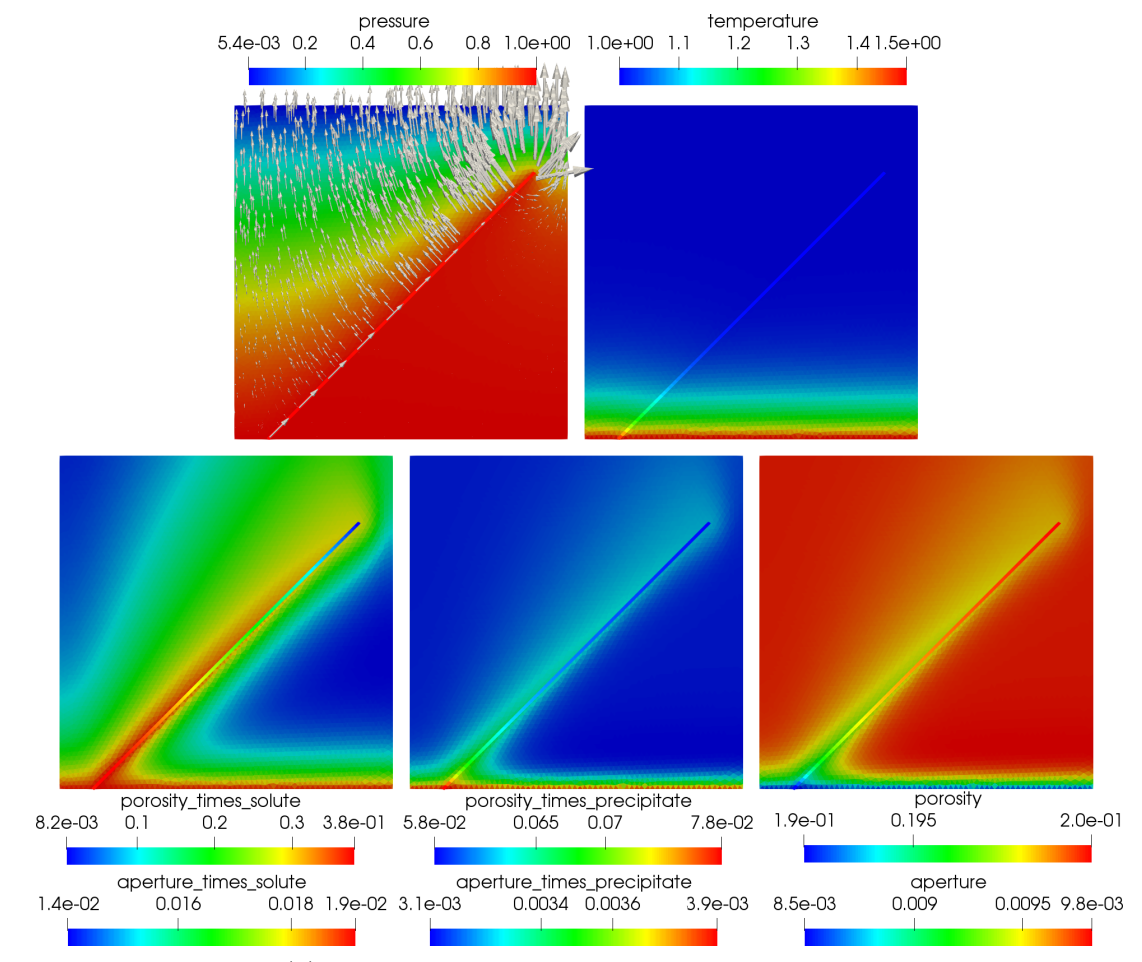

(a) Solution at time 0.05 and time step $n=1$.

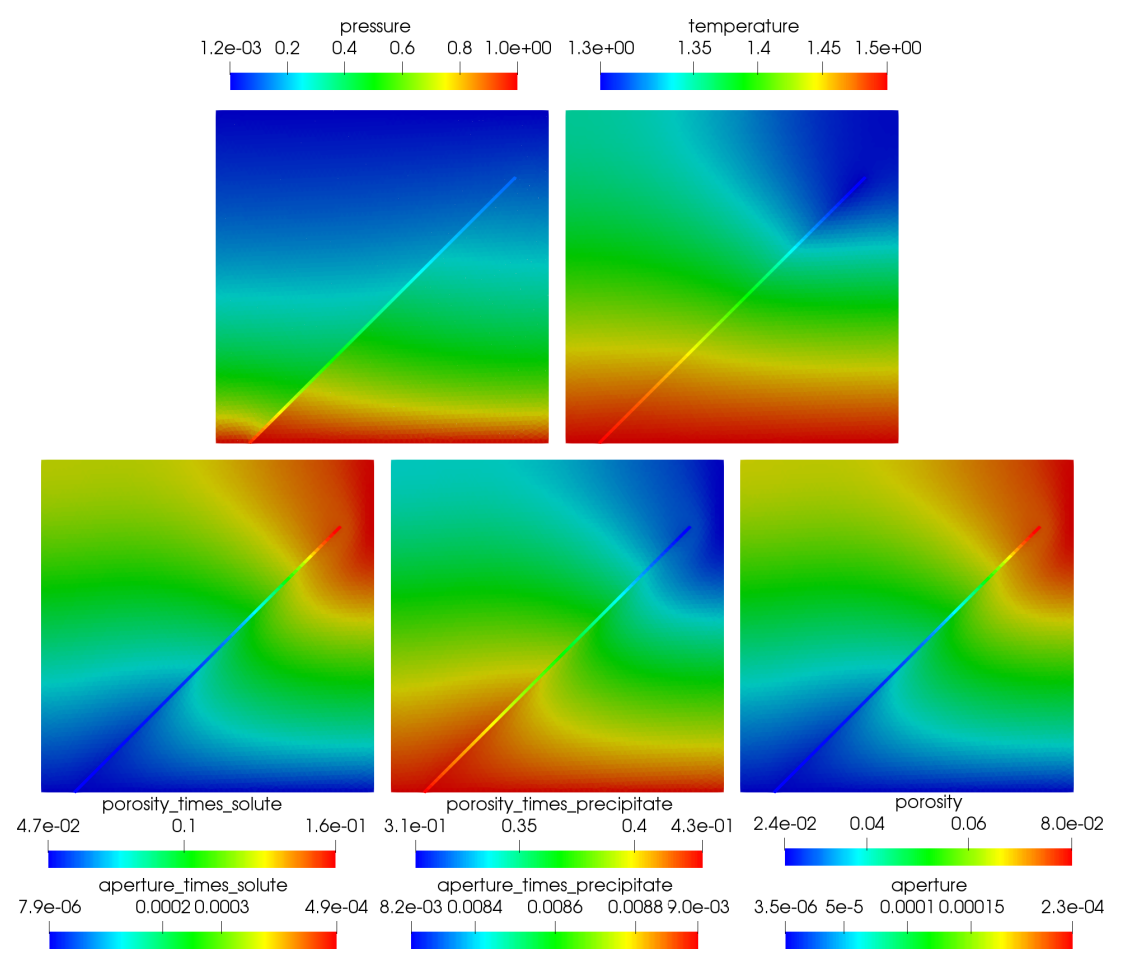

(b) Solution at time 2.25 and time step $n=45$.

Figure 11: Numerical solutions of the example in section 6.2.1 


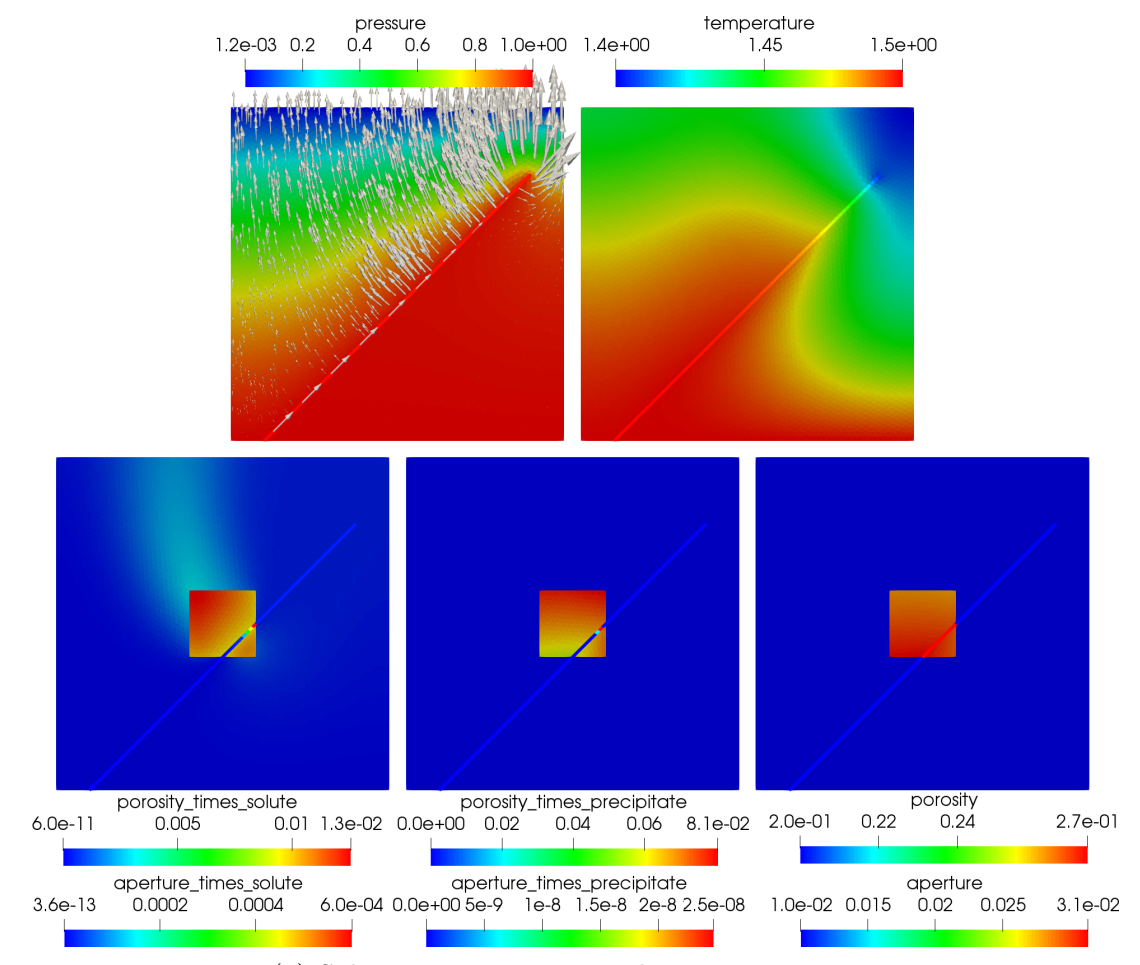

(a) Solution at time 1.15 and time step $n=23$.

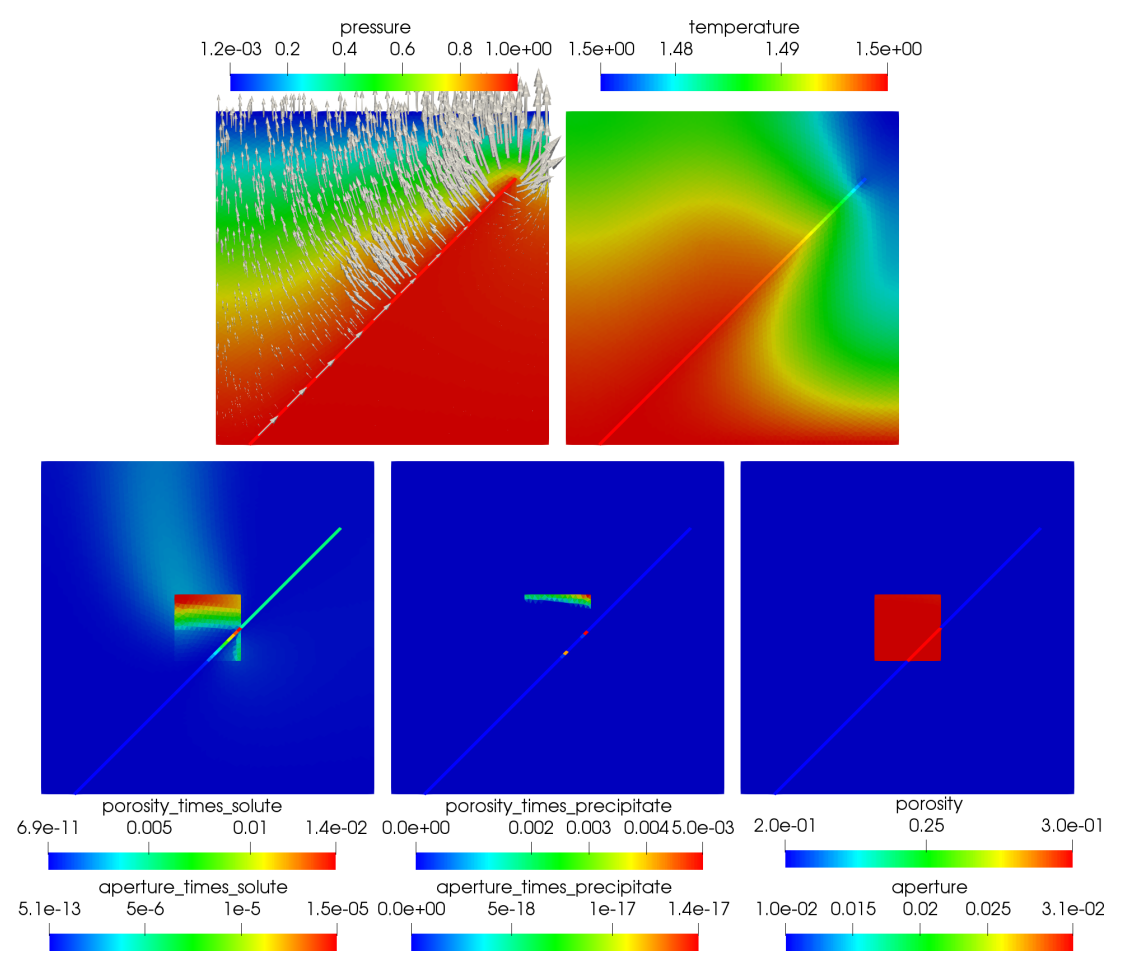

(b) Solution at time 1.55 and time step $n=31$.

Figure 12: Numerical solutions of the example in section 6.2.1. 

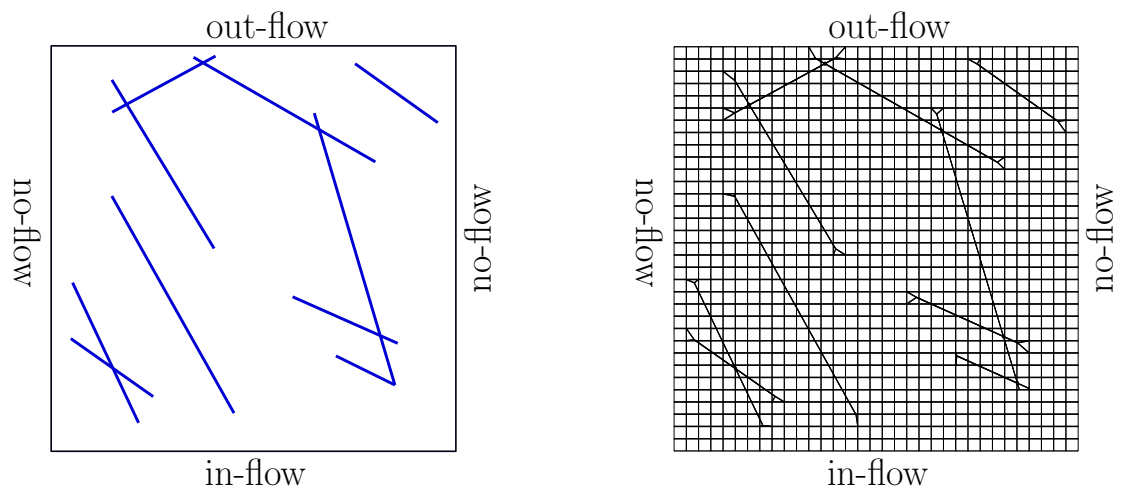

Figure 13: On the left, domain $\Omega$ and fracture $\gamma$ for the example of Subsection 6.3. On the right, the computation grid with the cut elements.

We consider two different settings, in the first the solute is injected from the bottom and starts to react while transported upward. In the second, the fractures are filled with precipitate and clean water is injected reacting and opening the fractures. The data are the same as in the previous case and reported in Table 2, with the only exception of $\eta_{\gamma}=4$ to emphasize aperture changes.

In this example the solution plots follow the same organization as before.

\subsubsection{Solute injection}

In this first case the additional data are the same as in section 6.2.1. The end time is now set as $T=2.5$ discretized with 50 time steps.

In this example, we inject solute from the bottom of the system along with hot water. Figure 14 reports the solutions obtained for different time steps. As the solute flows in the porous media and fractures it reacts forming new precipitate. The precipitate occludes the pores and attaches to the fracture walls, reducing the porosity and fracture aperture. The latter is reduced more rapidly since the fractures, in the beginning, are more permeable than the surrounding medium. At the second time step many fractures are already almost occluded with an impact on the Darcy velocity which drops and can barely detected.

This example shows the interaction between a complex network and the occlusion of the fractures. This in an interesting phenomena that the model is able to reproduce.

\subsubsection{Opening fractures}

In this case most of the additional data are the same as in section 6.2.2. However, now the fractures have aperture $\epsilon=10^{-4}$ with an initial precipitate $w_{\gamma, 0}=10$. In the porous media the initial precipitate is set to $w_{0}=0$.

The numerical solution of this example is represented in Figure 15 . We see that in the first time step shown, fractures still have small aperture in most parts, but it starts to increase as the high temperature front advances. At the same time the precipitate dissolves and new solute is created and transported upwards. In the second block of figures, most of the fractures have substantially increased their aperture and the precipitate is now only present at the top of the domain. Even if not represented, at the end of the simulation all the precipitate has reacted and all the solute has been transported away from the domain. The aperture reaches a stable value of approximately $4.5 \cdot 10^{-3}$ for all fractures. 


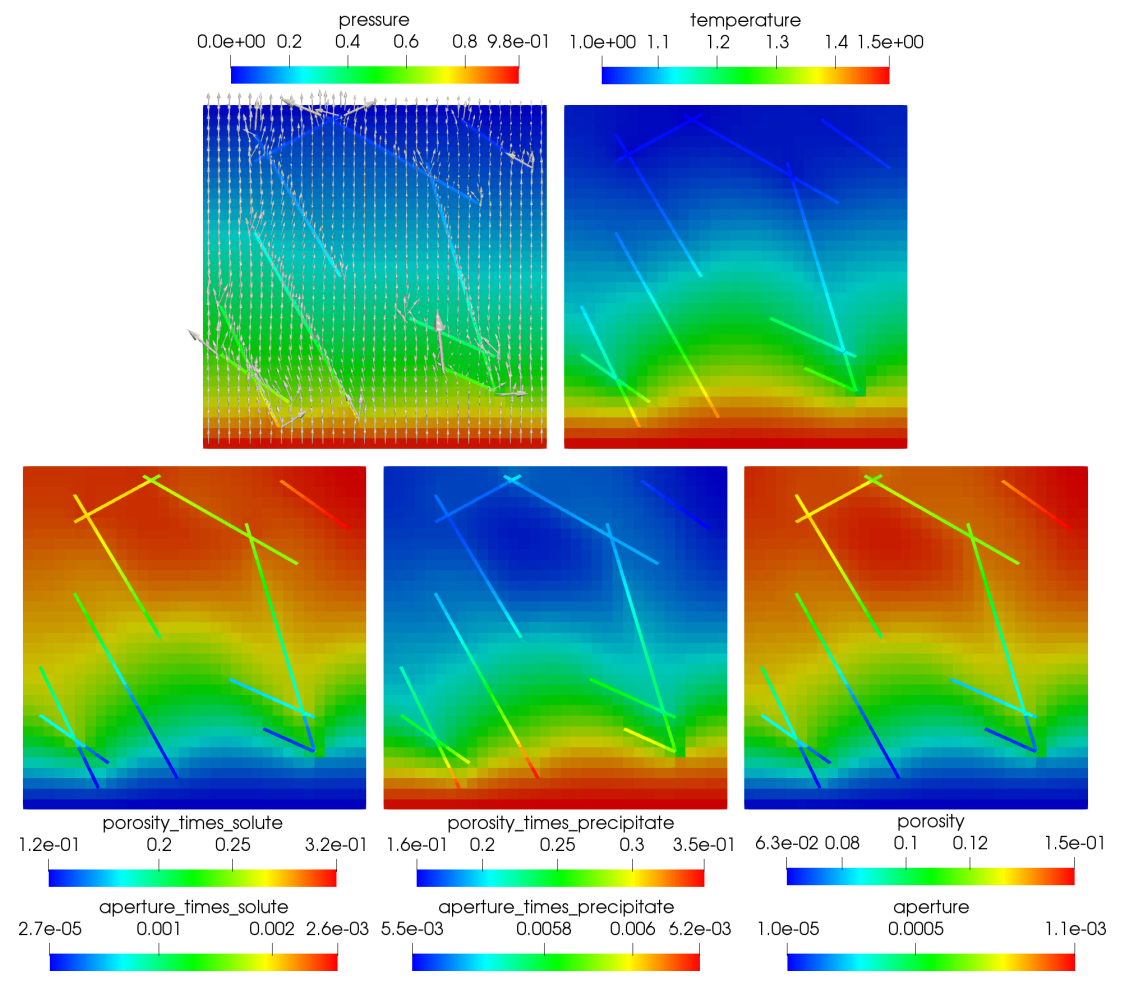

(a) Solution at time 1.25 and time step $n=25$.
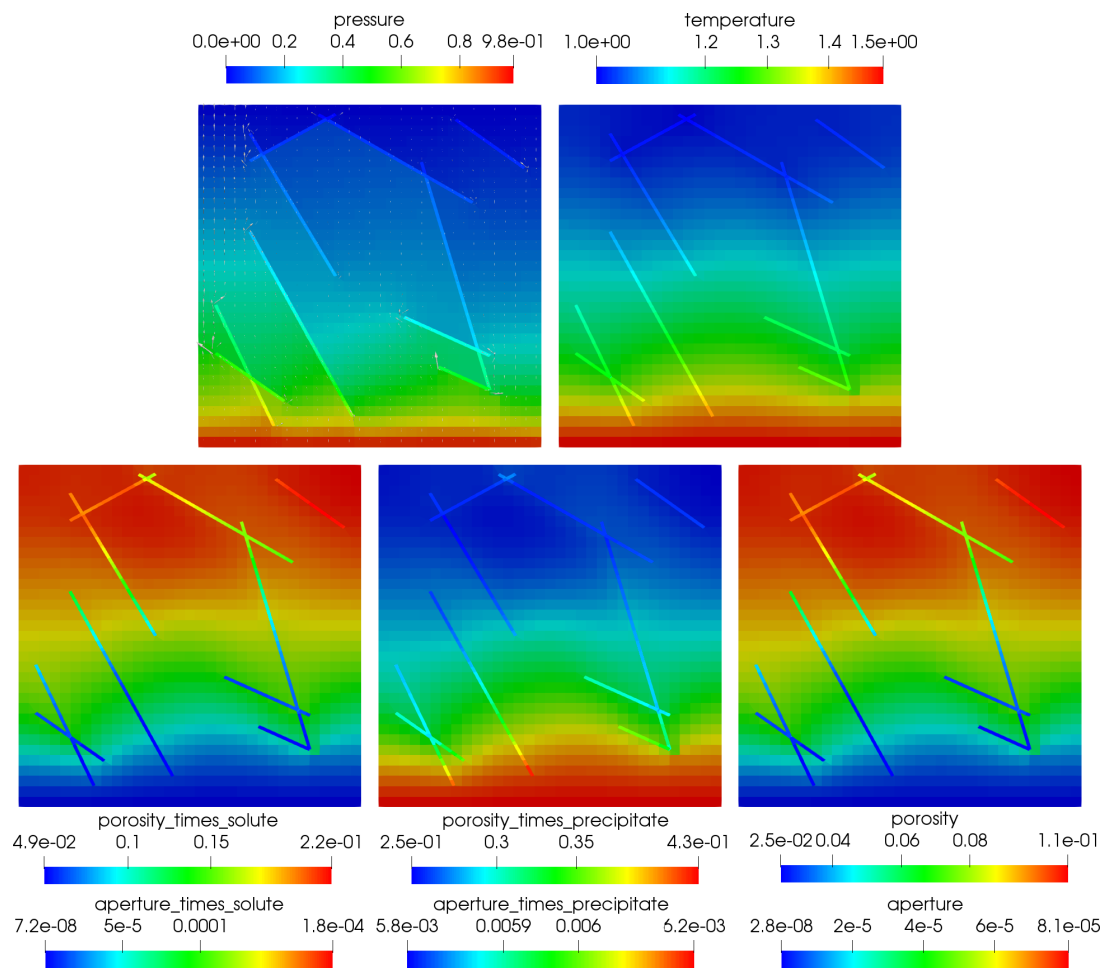

(b) Solution at time 2.25 and time step $n=45$.

Figure 14: Numerical solutions of the example in section 6.3.1. 


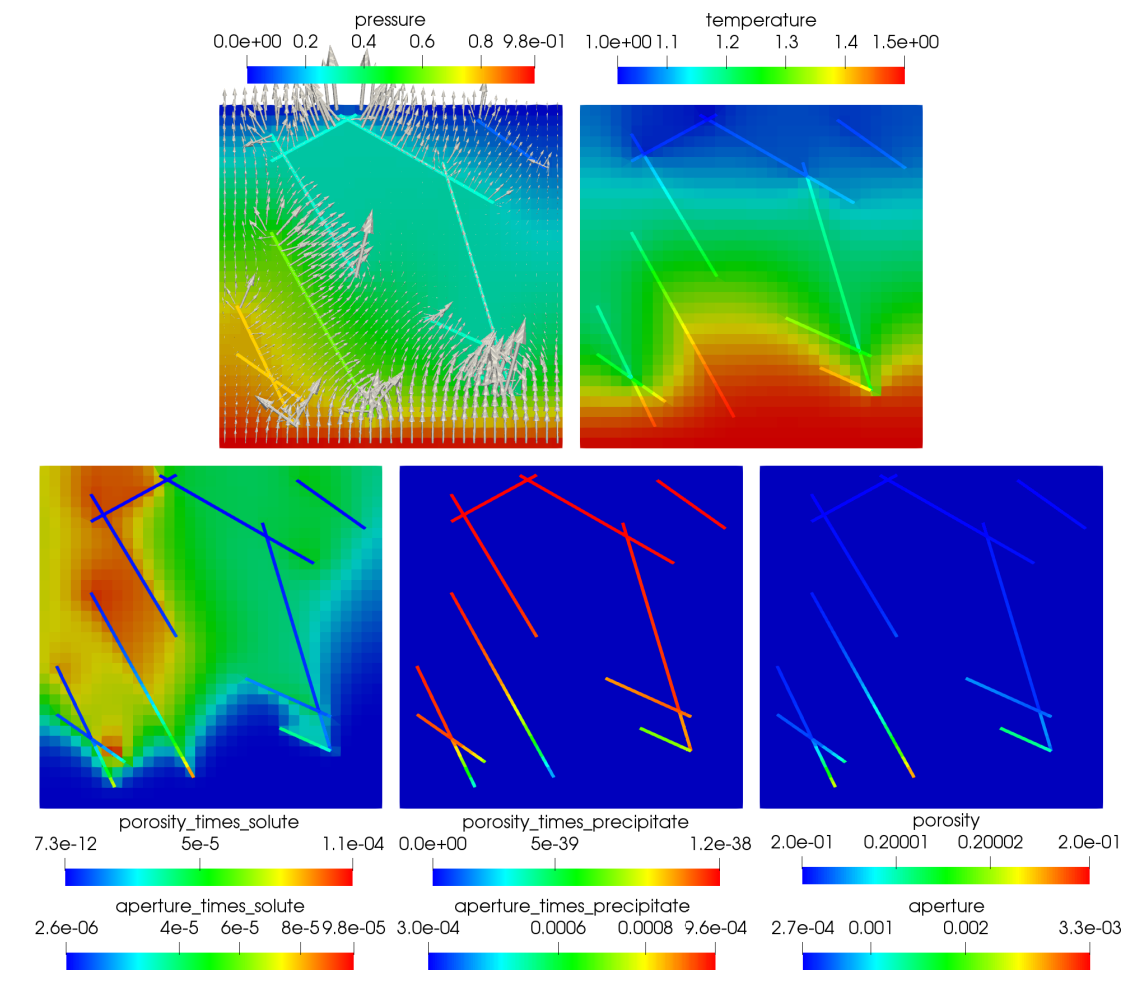

(a) Solution at time 1.3 and time step $n=26$.

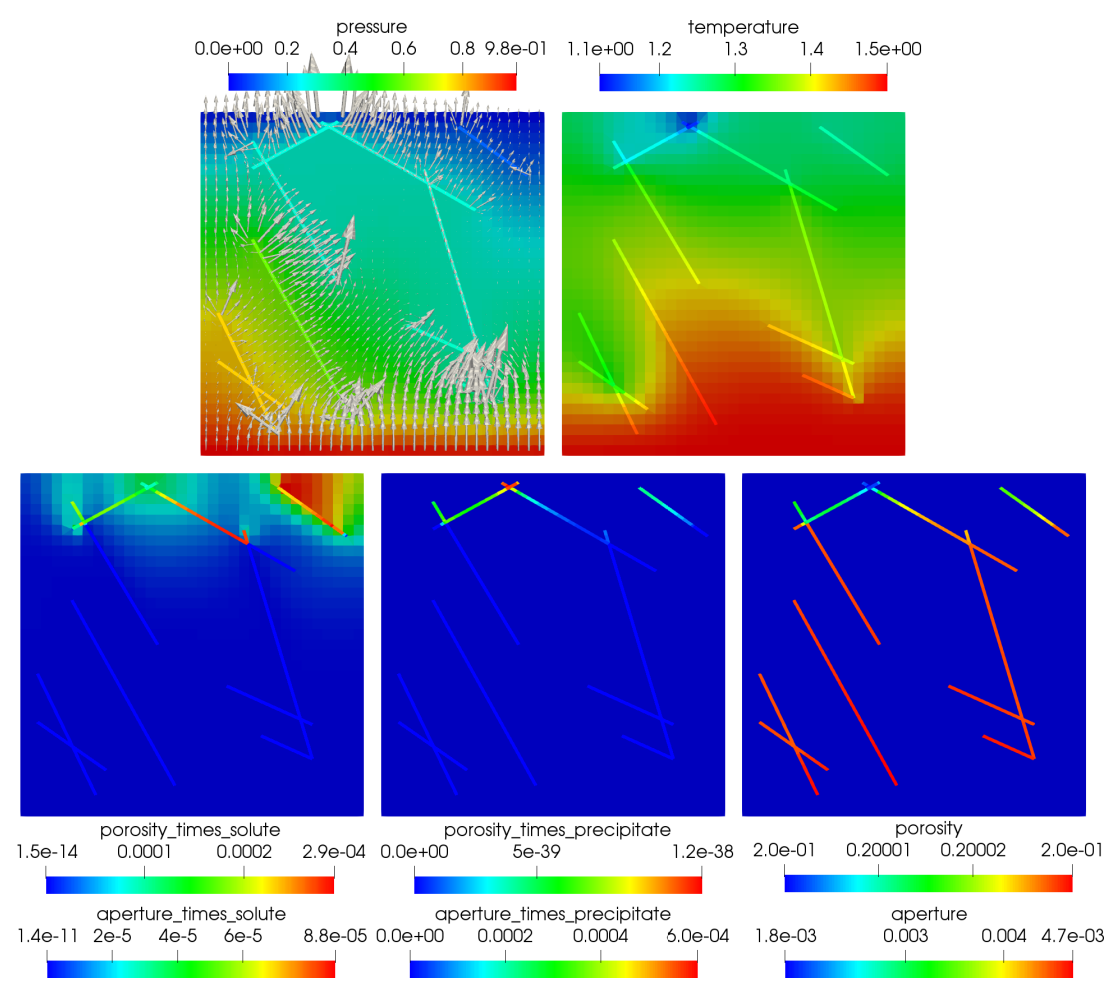

(b) Solution at time 3.25 and time step $n=65$.

Figure 15: Numerical solutions of the example in section 6.3.2. 
The possibility to open and thus enhance the flow property of a geothermal system is crucial in its exploitation. This simple example showed that the model is also able to capture such scenario.

\section{Conclusion}

In this work we have presented a mathematical model to describe in a fractured porous media a reactive single-phase flow with thermal effects. The mathematical model consists of a set of coupled PDEs and ODEs for the evolution of pressure, Darcy velocity, porosity, temperature, solute and precipitate concentration. Moreover, this model considers fractures as one co-dimensional manifolds in the framework of the so-called mixed-dimensional setting, so that fracture aperture is not any more a geometrical constraint but a model parameter and it can thus freely vary during the simulation. The resulting system is fully coupled, non-linear and generally non-smooth due to the modeling of the chemical reactions involved. To numerically solve the full system, we have introduced a temporal splitting scheme so that each physical process is solved sequentially but ensuring, as proven only experimentally by means of numerical tests, the mass conservation of the system. A nested splitting is used to compute the solute concentration separating the advective-diffusive parts from the reaction. For the latter a system of discontinuous ordinary differential equations is solved by using an event driven approach. To conclude, as the numerical examples showed the presented model is able to capture interesting and physically relevant phenomena also in presence of complex fracture networks.

\section{References}

[1] Abramo Agosti, Luca Formaggia, and Anna Scotti. Analysis of a model for precipitation and dissolution coupled with a darcy flux. Journal of Mathematical Analysis and Applications, 431(2):752-781, 2015.

[2] Abramo Agosti, Bianca Giovanardi, Luca Formaggia, and Anna Scotti. A numerical procedure for geochemical compaction in the presence of discontinuous reactions. Advances in Water Resources, 94:332 - 344, 2016.

[3] Elyes Ahmed, Alessio Fumagalli, and Ana Budiša. A multiscale flux basis for mortar mixed discretizations of reduced darcy-forchheimer fracture models. Computer Methods in Applied Mechanics and Engineering, 354:16-36, 2019.

[4] Elyes Ahmed, Jérôme Jaffré, and Jean E. Roberts. A reduced fracture model for twophase flow with different rock types. Mathematics and Computers in Simulation, 137:49-70, 2017. MAMERN VI-2015: 6th International Conference on Approximation Methods and Numerical Modeling in Environment and Natural Resources.

[5] Clarisse Alboin, Jérôme Jaffré, Jean E. Roberts, and Christophe Serres. Modeling fractures as interfaces for flow and transport in porous media. In Fluid flow and transport in porous media: mathematical and numerical treatment (South Hadley, MA, 2001), volume 295 of Contemp. Math., pages 13-24. Amer. Math. Soc., Providence, RI, 2002.

[6] Clarisse Alboin, Jérôme Jaffré, Jean E. Roberts, Xuewen Wang, and Christophe Serres. Domain decomposition for some transmission problems in flow in porous media, volume 552 of Lecture Notes in Phys., pages 22-34. Springer, Berlin, 2000. 
[7] Philippe Angot. A model of fracture for elliptic problems with flux and solution jumps. Comptes Rendus Mathematique, 337(6):425-430, 2003.

[8] Paola F. Antonietti, Chiara Facciolà, Alessandro Russo, and Marco Verani. Discontinuous galerkin approximation of flows in fractured porous media on polytopic grids. SIAM Journal on Scientific Computing, 41(1):A109-A138, 2019.

[9] Paola Francesca Antonietti, Luca Formaggia, Anna Scotti, Marco Verani, and Nicola Verzotti. Mimetic finite difference approximation of flows in fractured porous media. ESAIM: M2AN, 50(3):809-832, 2016.

[10] Jacob Bear. Dynamics of Fluids in Porous Media. American Elsevier, 1972.

[11] Jacob Bear and Yehuda Bachmat. Introduction to Modeling of Transport Phenomena in Porous Media. Theory and Applications of Transport in Porous Media. Springer Netherlands, 1990.

[12] Lourenço Beirão da Veiga, Franco Brezzi, Luisa Donatella Marini, and Alessandro Russo. Mixed virtual element methods for general second order elliptic problems on polygonal meshes. ESAIM: M2AN, 50(3):727-747, 2016.

[13] Matías Fernando Benedetto, Stefano Berrone, Andrea Borio, Sandra Pieraccini, and Stefano Scialò. A hybrid mortar virtual element method for discrete fracture network simulations. Journal of Computational Physics, 306:148 - 166, 2016.

[14] Matías Fernando Benedetto, Stefano Berrone, Sandra Pieraccini, and Stefano Scialò. The virtual element method for discrete fracture network simulations. Computer Methods in Applied Mechanics and Engineering, 280(0):135-156, 2014.

[15] Inga Berre, Wietse M. Boon, Bernd Flemisch, Alessio Fumagalli, Dennis Gläser, Eirik Keilegavlen, Anna Scotti, Ivar Stefansson, Alexandru Tatomir, Konstantin Brenner, Samuel Burbulla, Philippe Devloo, Omar Duran, Marco Favino, Julian Hennicker, I-Hsien Lee, Konstantin Lipnikov, Roland Masson, Klaus Mosthaf, Maria Giuseppina Chiara Nestola, Chuen-Fa Ni, Kirill Nikitin, Philipp Schädle, Daniil Svyatskiy, Ruslan Yanbarisov, and Patrick Zulian. Verification benchmarks for single-phase flow in three-dimensional fractured porous media. Technical report, arXiv:2002.07005 [math.NA], 2020.

[16] Inga Berre, Florian Doster, and Eirik Keilegavlen. Flow in fractured porous media: A review of conceptual models and discretization approaches. Transport in Porous Media, 130(1):215-236, 2019.

[17] Daniele Boffi, Franco Brezzi, and Michel Fortin. Mixed Finite Element Methods and Applications. Springer Series in Computational Mathematics. Springer Berlin Heidelberg, 2013.

[18] Wietse M. Boon, Jan M. Nordbotten, and Ivan Yotov. Robust discretization of flow in fractured porous media. SIAM Journal on Numerical Analysis, 56(4):2203-2233, 2018.

[19] Franco Brezzi, Richard S. Falk, and Donatella Luisa Marini. Basic principles of mixed virtual element methods. ESAIM: M2AN, 48(4):1227-1240, 2014.

[20] Florent Chave, Daniele A. Di Pietro, and Luca Formaggia. A hybrid high-order method for darcy flows in fractured porous media. SIAM Journal on Scientific Computing, 40(2):A1063A1094, 2018. 
[21] Florent Chave, Daniele A. Di Pietro, and Luca Formaggia. A hybrid high-order method for passive transport in fractured porous media. GEM - International Journal on Geomathematics, 10(1):12, 2019.

[22] Jean Côté and Jean-Marie Konrad. A generalized thermal conductivity model for soils and construction materials. Canadian Geotechnical Journal, 42(2):443-458, 2005.

[23] Jean-Raynald de Dreuzy, Géraldine Pichot, Baptiste Poirriez, and Jocelyne Erhel. Synthetic benchmark for modeling flow in 3d fractured media. Computers 63 Geosciences, 50:59 - 71, 2013. Benchmark problems, datasets and methodologies for the computational geosciences.

[24] Lopez L. Dieci L. Sliding motion in discontinuous differential systems: Theory and a computational apporach. 2008.

[25] Jérôme Droniou. Finite volume scheme for diffusion equations: Introduction to and review of modern methods, April 2013.

[26] Robert Eymard, Thierry Gallouët, and Raphaèle Herbin. Finite volume methods. In P. G. Ciarlet and J. L. Lions, editors, Solution of Equation in $\mathcal{R}^{n}$ (Part 3), Techniques of Scientific Computing (Part 3), volume 7 of Handbook of Numerical Analysis, pages 713-1018. Elsevier, 2000 .

[27] Isabelle Faille, Eric Flauraud, Frédéric Nataf, Sylvie Pégaz-Fiornet, Frédéric Schneider, and Françoise Willien. A New Fault Model in Geological Basin Modelling. Application of Finite Volume Scheme and Domain Decomposition Methods. In Finite volumes for complex applications, III (Porquerolles, 2002), pages 529-536. Hermes Sci. Publ., Paris, 2002.

[28] Isabelle Faille, Alessio Fumagalli, Jérôme Jaffré, and Jean Elisabeth Roberts. Model reduction and discretization using hybrid finite volumes of flow in porous media containing faults. Computational Geosciences, 20(2):317-339, 2016.

[29] Isabelle Faille, Frédéric Nataf, Laurent Saas, and Françoise Willien. Finite Volume Methods on Non-Matching Grids with Arbitrary Interface Conditions and Highly Heterogeneous Media. In Domain Decomposition Methods in Science and Engineering, volume 40 of Lecture Notes in Computational Science and Engineering, pages 243-250. Springer Berlin Heidelberg, 2005.

[30] Bernd Flemisch, Inga Berre, Wietse Boon, Alessio Fumagalli, Nicolas Schwenck, Anna Scotti, Ivar Stefansson, and Alexandru Tatomir. Benchmarks for single-phase flow in fractured porous media. Advances in Water Resources, 111:239-258, Januray 2018.

[31] Bernd Flemisch, Alessio Fumagalli, and Anna Scotti. A Review of the XFEM-Based Approximation of Flow in Fractured Porous Media, volume 12 of SEMA SIMAI Springer Series, chapter Advances in Discretization Methods, pages 47-76. Springer International Publishing, Cham, 2016.

[32] Luca Formaggia, Alessio Fumagalli, Anna Scotti, and Paolo Ruffo. A reduced model for Darcy's problem in networks of fractures. ESAIM: Mathematical Modelling and Numerical Analysis, 48:1089-1116, 72014.

[33] Najla Frih, Vincent Martin, Jean Elisabeth Roberts, and Ai Saâda. Modeling fractures as interfaces with nonmatching grids. Computational Geosciences, 16(4):1043-1060, 2012. 
[34] Najla Frih, Jean E. Roberts, and Ali Saada. Modeling fractures as interfaces: a model for Forchheimer fractures. Computers and Geosciences, 12(1):91-104, 2008.

[35] Alessio Fumagalli and Isabelle Faille. A double-layer reduced model for fault flow on slipping domains with hybrid finite volume scheme. SIAM Journal on Scientific Computing, 77:1-26, June 2018.

[36] Alessio Fumagalli and Eirik Keilegavlen. Dual virtual element method for discrete fractures networks. SIAM Journal on Scientific Computing, 40(1):B228-B258, 2018.

[37] Alessio Fumagalli and Eirik Keilegavlen. Dual virtual element methods for discrete fracture matrix models. Oil \& Gas Science and Technology - Revue d'IFP Energies nouvelles, 74(41):1-17, 2019.

[38] Alessio Fumagalli, Eirik Keilegavlen, and Stefano Scialò. Conforming, non-conforming and non-matching discretization couplings in discrete fracture network simulations. Journal of Computational Physics, 376:694-712, 2019.

[39] Alessio Fumagalli and Anna Scotti. A numerical method for two-phase flow in fractured porous media with non-matching grids. Advances in Water Resources, 62, Part C(0):454464, 2013. Computational Methods in Geologic CO2 Sequestration.

[40] Alessio Fumagalli and Anna Scotti. A Reduced Model for Flow and Transport in Fractured Porous Media with Non-matching Grids. In Andrea Cangiani, Ruslan L. Davidchack, Emmanuil Georgoulis, Alexander N. Gorban, Jeremy Levesley, and Michael V. Tretyakov, editors, Numerical Mathematics and Advanced Applications 2011, pages 499-507. Springer Berlin Heidelberg, 2013.

[41] Alessio Fumagalli and Anna Scotti. An Efficient XFEM Approximation of Darcy Flows in Arbitrarily Fractured Porous Media. Oil and Gas Sciences and Technologies - Revue d'IFP Energies Nouvelles, 69(4):555-564, April 2014.

[42] Alessio Fumagalli and Anna Scotti. Reactive flow in fractured porous media. In Finite Volumes for Complex Applications IX proceedings. Springer, 2020. Accepted.

[43] Alessio Fumagalli, Anna Scotti, and Luca Formaggia. Performances of the mixed virtual element method on complex grids for underground flow. Accepted in SEMA SIMAI Springer Series. Available at arXiv:2002.11974 [math.NA], 2020.

[44] Bianca Giovanardi, Anna Scotti, Luca Formaggia, and Paolo Ruffo. A general framework for the simulation of geochemical compaction. Computational Geosciences, 19(5):1027-1046, Oct 2015 .

[45] C.M. Guldberg and P. Waage. Studies concerning affinity. WileyBlackwell, 1864.

[46] Rainer Helmig. Multiphase flow and transport processes in the subsurface: a contribution to the modeling of hydrosystems. Springer-Verlag, Berlin, Germany, 1997.

[47] Jérôme Jaffré, Vincent Martin, and Jean E. Roberts. Generalized cell-centered finite volume methods for flow in porous media with faults. In Finite volumes for complex applications, III (Porquerolles, 2002), pages 343-350. Hermes Sci. Publ., Paris, 2002.

[48] Jérôme Jaffré, Mokhles Mnejja, and Jean E. Roberts. A discrete fracture model for twophase flow with matrix-fracture interaction. Procedia Computer Science, 4:967-973, 2011. 
[49] Eirik Keilegavlen, Runar Berge, Alessio Fumagalli, Michele Starnoni, Ivar Stefansson, Jhabriel Varela, and Inga Berre. Porepy: An open-source software for simulation of multiphysics processes in fractured porous media. Technical report, arXiv:1908.09869 [math.NA], 2019.

[50] Peter Knabner and Jean Elisabeth Roberts. Mathematical analysis of a discrete fracture model coupling darcy flow in the matrix with darcy-forchheimer flow in the fracture. ESAIM: Mathematical Modelling and Numerical Analysis, 48:1451-1472, 92014.

[51] Peter Knabner, C.J. van Duijn, and S. Hengst. An analysis of crystal dissolution fronts in flows through porous media. part 1: Compatible boundary conditions. Advances in Water Resources, 18(3):171-185, 1995.

[52] Vincent Martin, Jérôme Jaffré, and Jean Elisabeth Roberts. Modeling Fractures and Barriers as Interfaces for Flow in Porous Media. SIAM J. Sci. Comput., 26(5):1667-1691, 2005.

[53] Fernando Morales and Ralph E. Showalter. The narrow fracture approximation by channeled flow. Journal of Mathematical Analysis and Applications, 365(1):320-331, 2010.

[54] Fernando Morales and Ralph E. Showalter. Interface approximation of darcy flow in a narrow channel. Mathematical Methods in the Applied Sciences, 35(2):182-195, 2012.

[55] Jan Martin Nordbotten, Wietse Boon, Alessio Fumagalli, and Eirik Keilegavlen. Unified approach to discretization of flow in fractured porous media. Computational Geosciences, 23(2):225-237, 2019.

[56] Jan Martin Nordbotten and Micheal A. Celia. Geological Storage of CO2: Modeling Approaches for Large-Scale Simulation. Wiley, 2011.

[57] Pierre-Arnaud Raviart and Jean-Marie Thomas. A mixed finite element method for second order elliptic problems. Lecture Notes in Mathematics, 606:292-315, 1977.

[58] Jean E. Roberts and Jean-Marie Thomas. Mixed and hybrid methods. In Handbook of numerical analysis, Vol. II, Handb. Numer. Anal., II, pages 523-639. North-Holland, Amsterdam, 1991.

[59] Tor Harald Sandve, Inga Berre, and Jan Martin Nordbotten. An efficient multi-point flux approximation method for Discrete Fracture-Matrix simulations. Journal of Computational Physics, 231(9):3784-3800, 2012.

[60] Nicolas Schwenck, Bernd Flemisch, Rainer Helmig, and BarbaraI. Wohlmuth. Dimensionally reduced flow models in fractured porous media: crossings and boundaries. Computational Geosciences, 19(6):1219-1230, 2015.

[61] Ivar Stefansson, Inga Berre, and Eirik Keilegavlen. Finite-volume discretisations for flow in fractured porous media. Transport in Porous Media, 124(2):439-462, Sep 2018.

[62] Xavier Tunc, Isabelle Faille, Thierry Gallouët, Marie Christine Cacas, and Pascal Havé. A model for conductive faults with non-matching grids. Computational Geosciences, 16:277296, 2012.

[63] Tycho L. van Noorden. Crystal precipitation and dissolution in a porous medium: Effective equations and numerical experiments. Multiscale Modeling $\& 3$ Simulation, 7(3):1220-1236, 2009 . 